\title{
What is Data? - Exploring the Meaning of Data in Data Physicalisation Teaching
}

\author{
Rosa van Koningsbruggen \\ Bauhaus-Universität Weimar \\ Germany \\ rosa.donna.van.koningsbruggen@uni- \\ weimar.de
}

\author{
Hannes Waldschütz \\ Bauhaus-Universität Weimar \\ Germany \\ hannes.waldschuetz@uni-weimar.de
}

\author{
Eva Hornecker \\ Bauhaus-Universität Weimar \\ Germany \\ eva.hornecker@uni-weimar.de
}

\begin{abstract}
A growing body of work focuses on physicalisations based on personal, everyday data. Despite growing interest, little is known about how to educate people on their creation. We designed a teaching method of 'Data Diaries', which consists of five representation assignments that move from visualising to physicalising personal data. The Data Diaries were used in a semester project, with the aim of creating an interactive physicalisation. We analysed the Data Diaries, written reports, and participant interviews. Our analysis shows that people need to overcome the challenge of using materiality to communicate data, which happens in four stages. Moreover, the materiality made participants realise that physicalisations do not focus on efficiency and accuracy, but on the story of the data, by referring to its origin, use of personal mappings, and reduction. As physicalisations blur the line between quantitative and qualitative, designing them engenders a change in our notion of 'data'.
\end{abstract}

\section{CCS CONCEPTS}

- Human-centered computing $\rightarrow$ Empirical studies in $\mathrm{HCI}$; HCI theory, concepts and models.

\section{KEYWORDS}

Data Diaries, Notion of Data, Physicalisation Literacy, Teaching

\section{ACM Reference Format:}

Rosa van Koningsbruggen, Hannes Waldschütz, and Eva Hornecker. 2022. What is Data? - Exploring the Meaning of Data in Data Physicalisation Teaching. In Sixteenth International Conference on Tangible, Embedded, and Embodied Interaction (TEI '22), February 13-16, 2022, Daejeon, Republic of Korea. ACM, New York, NY, USA, 21 pages. https://doi.org/10.1145/3490149. 3501319

\section{INTRODUCTION}

An increasing body of work concerns itself with the physicalisation of personal data, e.g. [3, 10, 31]. Here, data is encoded through the material and geometric properties of physical artefacts [28]. Such physicalisations have been shown to let the user "feel their data" [39] and foster self-reflection [30, 57]. Moreover, they appear to trigger deeper connections to data (not just regarding personal

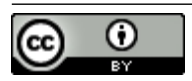

This work is licensed under a Creative Commons Attribution International 4.0 License.

TEI '22, February 13-16, 2022, Daejeon, Republic of Korea (c) 2022 Copyright held by the owner/author(s). Publication rights licensed to ACM. ACM ISBN 978-1-4503-9147-4/22/02.

https://doi.org/10.1145/3490149.3501319 data, but data in general) than visualisations [23, 62] and seem to be especially suited for representing personal, everyday data. One of their biggest strengths is their material nature, which helps to circumvent the imaginaries that data are immaterial, digital, and objective $[10,13,24]$. By mapping data to an artefact which is actually graspable, the data are brought into our physical world, where separating them from the representation is nearly impossible [46]. Their physicality also helps to highlight the connectedness, situatedness, and messiness of data [46,47], evading the perpetuation of data as something which is smooth and easily manipulable [17, 24]. Furthermore, physicalisations can trigger emotional responses [22, 62], enhance our engagement with data, and lower the cognitive load of analysing data $[28,56]$. Because of these advantages, data physicalisation has a lot of potential for representing personal, everyday life data, which is inherently messy, connected, emotional, and complex [10, 39].

However, data physicalisation brings new challenges with it as well. As put by Offenhuber: "[it] brings data from the unambiguous symbolic space into the real world, where data is a more complicated affair" [47]: the context of the representation plays a role and many aspects, such as the light fall on the object, are outside the designer's control. Moreover, one has to combine technical, material, and artistic skills and knowledge to create good data physicalisations [28]. This combination of competencies seems difficult to embody: technical scholars mostly focus on creating dynamic data physicalisations based on standard visual representations (graphs), such as EMERGE [56] and inFORM [15]. Although these communicate data through a physical artefact, their aesthetics and interaction do not take advantage of the 3D- and rich material nature of physicalisations, contrary to the physicalisations created by designers (e.g. $[11,47,55])$.

Therefore, our starting question was how to teach creators the designerly skills to be able to explore physicalisation's new representation opportunities. In particular, how can we foster a mindset which enables people to understand and take advantage of the richness that physicalisations allow for? We discuss our method called 'Data Diaries': a set of weekly data representation assignments inspired by Lupi and Posavec's book "Dear Data" [38]. The Data Diaries moved from visualising personal, everyday data to physicalising it, as such making participants aware of the data surrounding them, whilst challenging them to develop the needed skills, and to expand their perspective of what data is. At the end of this semester project, participants then created an interactive data physicalisation of self-chosen personal data.

Looking at participants' work over these weeks, we noticed interesting developments, which motivated a detailed analysis and 
reflection. We analysed all submissions, identifying themes across participants and Data Diaries, development over time, including participants' written reports. We further interviewed half the participants to deepen our insights.

Our analysis reveals that in order to learn how to use materiality to represent data, participants went through four learning stages. In the first stage, participants feel overwhelmed by the idea of representing data through a material artefact. In the second stage, participants use their knowledge of visualisation for the creation of physicalisations. However, as they realise this does not work, they move to the third stage. Here they discover that everything can be used to physicalise your data. In the fourth and final stage, participants realise that there should be a symbiosis between data, method of collection, and material, in order to tell the story to be represented.

The main insight of our work is an understanding of our notion of data and how this needs to be expanded in order to design physicalisations. Through the Data Diaries, participants discovered that physicalisations do not adhere to the rules of visualisation, such as a focus on efficiency, accuracy, and neutrality [33]. Instead of making data seem objective [33] and representing it efficiently, physicalisations highlight the subjectivity of data, and represent them in a way which makes the user relate and connect. Data physicalisations were considered a human way of representing data, and blurred the line between what we consider quantitative and qualitative. As participants experienced the subjectivity of data and explored its qualitative aspects, their initial notion of data as an objective and neutral entity did not hold up anymore. Therefore, participants had to change their notion of data, something which was both demanded and facilitated by the creation of data physicalisations.

The contribution of our work is two-fold: Firstly, we provide insights into which elements can support the creation of physicalisation literacy. Secondly, we highlight that we need to expand our notion of data in order to design data physicalisations. We also show how the Data Diaries and physicalisation design trigger a rethinking - indicating that this may be fruitful as a more general approach for discussing 'what data is'. With this, we contribute to an emerging body of work which states that we need to reconsider what data is and how we understand it.

\section{RELATED WORK}

\subsection{Data Literacy and Data Physicalisation}

Due to data's increasing importance in society, the urgency to have a certain level of data literacy has increased as well. Data literacy includes "the abilities to select, clean, analyse, visualise, critique and interpret data, as well as to communicate stories from data and to use data as part of a design process" [66]. Akin to data literacy is the concept of visualisation literacy, which entails the ability to create and interpret visual data representations [5].

Previous work in Human Computer Interaction (HCI) (e.g. [2, $18,63,67]$ ) has uncovered various approaches in developing data and visualisation literacy. One approach used and suggested is scaffolding, e.g. [7, 8, 34]. Here, students receive supports, which guide and assist them in carrying out tasks that are initially too complex for them to independently complete [60]. The actual task is divided into smaller challenges, which help students to develop the needed knowledge and skills. Scaffolding has been used to help students form hypotheses, collect data, and analyse it [37, 43, 50]. Scaffolding might also be used to teach students how to work with the complexity and messiness of data [34].

Moreover, an emerging body of work focuses on the use of personal data [19, 29, 54]. This approach is based on the idea that people have a vast understanding of their own daily lives and activities, which enables them to reflect on, reason, and discuss patterns, correlations, and variations in their own data sets [36].

However, the traditional idea of data literacy does not suffice for personal data and physicalisations. As explained by Lupton [39], data literacy traditionally focuses on how people interpret and evaluate the validity of data, and how well they can assess and analyse digital data sets. However, for personal data, one has to account for the user's experience of generating that data as well, and how their experience affects how they understand and analyse their data. Similarly, data physicalisations demand new skill sets from the creator. Besides having a basic level of data literacy, physicalisation creators should have an understanding of design, materials, and various technical skills, such as programming and creating mechanisms [28].

As scaffolding works to teach students about complex problems and it is even suggested to help students learn about data's complexity [34], we explore how a scaffolding approach can help students acquire the designerly (and some technical) skills needed to create physicalisations and to develop a level of physicalisation literacy, in particular, understanding what is specific about the nature of physicalisations.

\subsection{Teaching Data Physicalisation}

At the time of writing this paper, there is only very limited work on how to teach people about the design of data physicalisations. The examples to be found are a workshop, and methods designed for workshops and user studies [21, 25, 57]. The aforementioned workshop explored the pedagogy of physicalisation, including what learning scenarios could benefit from using physicalisations and different approaches to introduce physicalisation activities to different learning audiences [21]. Huron et al. designed a workshop method which helps people to rapidly engage with and create simple physicalisations [25]. Key to this is a set of cards which provide participants with a constrained design space for the design of a data physicalisation. 'Activity cards' evoke an abstract purpose (such as "stimulate") for the physicalisation, 'scenario cards' set the scenario, and 'data cards' provide a data set. This reduces the initial hurdle of deciding on a data source, purpose, and environment for the physicalisation. Lastly, Thudt et al. guided participants towards creating a personal physicalisation in their domestic lives through an introductory workshop where they learn the basics of working with data and how to map it to visual variables [57]. Furthermore, the website dataphys.org has an overview section of (mostly) nonpublished teaching materials used for physicalisation courses [64]. These range from an assignment on "Personal Data Physicalization" to literature.

The above mentioned cases show there is a need for an understanding on how to teach physicalisation. Moreover, the examples 
provide insights in how people can be guided and prepared for the creation of physicalisations. However, what remains unexplored is how to help learners develop the designerly and conceptual skills needed, and how to make them aware of the different modalities, such as haptics and sound, that can be utilised in physicalisations. This is what our method of the Data Diaries aims to achieve.

\subsection{Personal Data}

Increasingly, we have become data subjects through our interactions with the technologies that surround us [40]. Friendships, bodies, habits, interests, and many more elements from our personal, daily lives are now being quantified: a process which can be referred to as datafication [44]. Personal datafication happens both consciously - for example the Quantified Self movement - or unwittingly. In the case of the former, personal data are used to optimise the user's life and to improve self-knowledge [10, 40, 40]. By quantifying our everyday life, the data can be understood from a birds-eye view: "from far away" [51]. This distance between our lived experience and portrayed information results in the belief that data is objective and neutral [51]. As such, we try to make our lives objective.

However, quantification omits important elements of data. Whereas quantification provides the illusion that data is placeless and universal, data is actually embedded in lively assemblages with other data, objects, and people [14, 39, 40]. Furthermore, quantification prevents emotional connection to the data [13], even though it is known that our senses and emotions play an integral role in knowledge retrieval and sense-making [16, 26]. Besides, an objectified perspective tends to hide the decisions that underlie which data is being collected and represented, and what ambiguities are hidden. To fully understand our data, we thus need to account for our ongoing reflexivity, emotional connection, and entanglement with data $[9,32]$.

Therefore, the Data Diaries used in our work focus on both the quantitative and qualitative: guiding participants to explore a fuller picture of their data. Moreover, we made the decision to not only focus on elements which are already being datafied (such as step count). Instead, we follow the following definition: "Personal data are any piece of information that can identify or be identifiable to an individual" [49].

\section{PROCESS AND TEACHING APPROACH}

The work presented here centres around the online student project "Data Perspectives. Physical Representations of Everyday Data" given at Bauhaus-Universität Weimar. The project was part of the university's project-based learning (PBL), where students explore a practical or research project in a project group. The aim of our project was to teach students about physicalisations and how to design for it, using personal, everyday data. Participants started the project with the five Data Diary assignments. These, together with discussion of literature and participants' work on their final interactive physicalisation, were discussed during weekly (online) seminars. Since the pandemic required us to teach online and distancing rules were recommended, participants worked on smaller, individual projects, instead of generating a shared larger outcome.
Eight participants (all students at the Bauhaus-Universität Weimar, of which one exchange student) took part in the project, after they had selected it as their semester-project. Six had a Computer Science background, the others were a Product Design and a Media Art student, of whom the latter had been working as an artist for nine years. Participants were aged between twenty and thirty-five years and had various nationalities (four German, one Swedish, one American, one Afghani, one Taiwanese). Five participants were in their Bachelor studies and three in their Master's.

Based on previous experience teaching PBL-projects on physicalisation, we knew that students have difficulties deciding on a data source and how to represent the data. To help students overcome these obstacles, we created five introductory assignments, the Data Diaries. These are a set of assignments, inspired by the didactic notion of scaffolding and the book "Dear Data". Its two authors sent each other weekly postcards for the duration of one year and document these in the book [38]. The postcards contained creative and artistic visualisations of personal data experienced, with weekly changing topics. The aim of our Data Diaries was to familiarise participants with creating data representations, introduce the different modalities of physicalisations (e.g. haptics and movements), help them develop the skills needed to create a data physicalisation, and sensitise them for the everyday data around them. This knowledge was then combined in the final task, where participants were asked to design an interactive physicalisation, which represents data from their personal lives. These hands-on assignments were guided by the seminars, where students presented their work, discussed physicalisation literature, e.g. [1, 27, 28, 30, 31, 45, 52, 55, 61], and received electronics tutorials covering basic Arduino principles and presentations given by the authors of this paper on design, design processes, data, and data physicalisation and representations. An overview of the curriculum can be found in Appendix A.

In total, we developed five Data Diary assignments, each to be conducted in a week. For each, participants received a challenge regarding personal data, which they had to track for the duration of a working week and represent. Participants then presented their results to us and each other during the seminar, and received feedback. At first, these representations focused on visualising data, but soon moved to the creation of physicalisations. The visualisations were not allowed to be standard visualisations, such as pie and bar charts. Instead, participants had to come up with their own creative and artistic way of representing data, just as in "Dear Data". During the physicalisation assignments, participants were challenged to explore haptics and movement, with the intent to sensitise them for the material nature of physicalisations and the possibility of interactive physicalisations. Through the Data Diaries we wanted to constrain participants' decision space, yet offer them freedom to make their own unique decisions in what and how to track the data. Table 1 shows an overview of the Data Diaries.

The five Data Diaries alternate between more constrained and open assignments. For the first week, we picked a data source which is all around us and relatively easy to quantify. By asking participants to track and visually represent the number of hellos and goodbyes, or compliments and apologies, we hoped that participants would ease into tracking elements of their personal lives and the idea of representing data in a creative way, without falling back on standard visualisation methods. The second week focused 
Table 1: Overview of the Data Diary assignments and the modality that had to be used.

\begin{tabular}{llllll}
\hline & Data Diary 1 & Data Diary 2 & Data Diary 3 & Data Diary 4 & Data Diary 5 \\
\hline Topic & $\begin{array}{l}\text { Number of hellos } \\
\text { and goodbyes } / \text { com- } \\
\text { pliments and apolo- } \\
\text { gies }\end{array}$ & Routes and paths & $\begin{array}{l}\text { Counting and Cate- } \\
\text { gorising }\end{array}$ & $\begin{array}{l}\text { Senses and Sensa- How I spent my time } \\
\text { tions }\end{array}$ \\
Visualisation & Visualisation & Physicalisation & $\begin{array}{l}\text { Physicalisation us- Physicalisation } \\
\text { ing tactile modality } \\
\text { using movement }\end{array}$ \\
\hline
\end{tabular}

on routes and paths: a slightly more abstract topic, but carrying a strong (visual) connotation. Here, we wanted to challenge participants to reinterpret the data source and how to represent it. After creating two visualisations, the required mode of representation was physicalisation. To help participants get used to working with physical artefacts, we selected a topic which could easily be represented through tangible objects: counting and categorising things. Next, as participants had gained experience with tracking data and created their first physicalisation, we challenged them to explore a more complex data source, which is not as easy to datafy, by asking them to represent their experience of senses and sensations. Suitably to the topic, participants had to represent their data using the tactile modality, to make them aware of the various modes which data physicalisation can use to represent data [28]. Finally, the fifth Data Diary had to communicate data via movement. As we believed movement to be the most abstract modality, the topic of this assignment was slightly easier than that of the fourth Data Diary: how participants spent their time. For the final task, participants then had to select a data source of their own and individually create an interactive data physicalisation.

\subsection{Analysis Approach}

In this paper, we discuss the outcomes of the Data Diaries and created physicalisations. Our findings and observations originate from three different sources: 1) images of the created Data Diaries and interactive physicalisations, 2) written reports about and documenting the design process, and 3) interviews with four participants on how they experienced the Data Diaries. When informed about our desire to write a paper about the project and whether the participants wanted to take part in the interview, none objected to their data being analysed and presented for publication purposes.

The images were analysed by three researchers, using open coding. The written reports were analysed by two researchers, who used thematic analysis to deduce themes [6]. Based on the findings of this analysis, participants were invited to take part in an individual interview. Four participants (two Bachelor and one Master HCI student, and one Master Media-Art student) agreed to take part and were asked about: 1) their journey throughout the project, 2) which Data Diary they learned the most from, 3) which they learned the least from, 4) what they learned about data from the Data Diaries, 5) what they learned about themselves from the Data Diaries, 6) how they would describe data now, 7) whether this used to be different and if so how, 8) what caused this difference, 9) what they believe the differences between creating a visualisation and physicalisation are, 10) what they learned about data from creating a physicalisation, and 11) what they learned about themselves from creating a physicalisation.

The interviews were audio-recorded, transcribed, and again analysed by two researchers using thematic analysis [6]. Following an inductive approach, the analysis happened in two stages. In the first stage, both researchers independently selected codes. These codes were then discussed one by one, to ensure both researchers agreed. The selected codes were then used to create the initial fourteen themes. After a period of reflection, the researchers checked whether they believed the codes were still assigned to the correct theme. Furthermore, the number of themes was reduced to twelve and their naming was finalised. Lastly, the researchers distinguished between five themes and seven sub-themes, and created a thematic map.

\section{JOURNEY THROUGHOUT THE DATA DIARIES}

To start, we highlight and discuss the journey participants experienced throughout the Data Diaries, how they developed throughout the assignments, and the main characteristics and changes across the series of Data Diaries. We do so by highlighting the data representations that embodied the experienced developments or direction(s) participants followed that week. An overview of all created Data Diaries can be found with the supplemental materials.

Participants are referred to as $P 1$ to $P 8$. If a quote is from the written report, this is indicated via a $-R$ behind the participant ID (e.g. P1-R). Quotes without $-R$ stem from the interviews. Report quotes are verbatim and unchanged.

\subsection{Data Diary 1: Hellos and Goodbyes / Compliments and Apologies}

For the first Data Diary, participants were asked to track either the number of hellos and goodbyes they sent or received, or the number of compliments and apologies. Although participants were free to pick either of the topics, seven decided to go for the Hellos and Goodbyes. Participants were challenged to not fall back on standard visualisation methods, which proved to be a challenge: "The idea of visualising data in another way but graphs is still very abstract" (P2-R). Nonetheless, participants succeeded in finding new ways to represent data. By using symbols and icons (as done by P6), participants captured elements such as who greeted them, where / via what platform, and how this made them feel, resulting in visualisations with multi-layered information. 


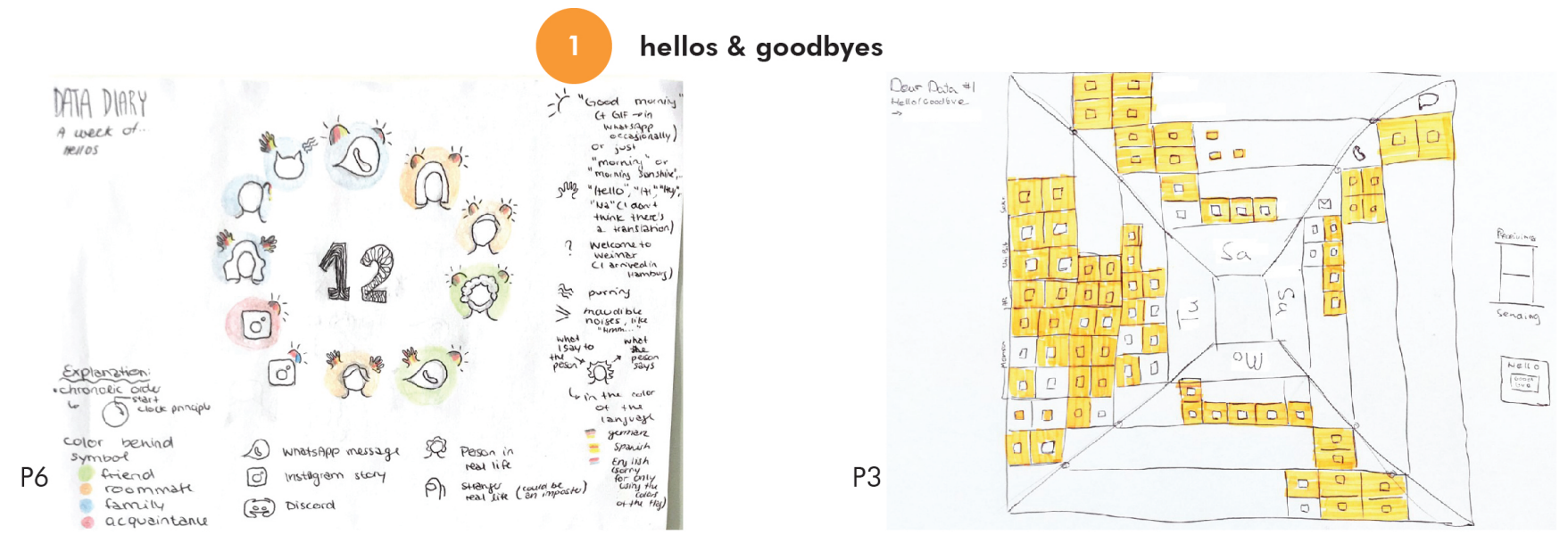

Figure 1: Two Data Diaries created for the first assignment. Left: P6 created a rich narrative visualisations representing who, when, where, and via what medium said hello or goodbye (including a purring cat), with colour coding of the type of relationship, the language used etc. Right: P3 created an analytical visualisation indicating whether an exchange of verbal greetings was complete or incomplete, marking hello's and goodbyes (outer vs. inner box) and P3 as receiver (top box in a row) or as 'sender' (lower box in a row).

Figure 1 illustrates two main approaches: analytical, abstract visualisations (exemplified by $\mathrm{P} 3$ ) and iconic-style, narrative visualisations (P6). P3 utilised their data to show whether a greeting was complete (as in two hellos and two goodbyes), or incomplete, for example only a 'hello' or 'goodbye'. P6, on the other hand, explored when they encountered a greeting (position on the "clock"), their relationship to that person or animal (colour), the language, and medium or location (online or IRL). The analytical visualisations reflect how participants thought of data at this point: "This visualization did also reflect well, how I percepted (sic.) data until then. Namely as a very mathematical and objective abstraction of processes and its outcomes in the reality" (P3-R). Analytical (P2, P3, P4, P8) versus more narrative approaches $(\mathrm{P} 1, \mathrm{P} 5, \mathrm{P} 6, \mathrm{P} 7)$ were distributed half-half.

\subsection{Data Diary 2: Routes and Paths}

For the second week (see Figure 2), participants were asked to visualise the routes and paths they took that week. What they considered a route or path was up to them to decide. This assignment resulted in mostly abstract visualisations, which obscured the exact locations of the designer. This led to vivid discussions during class, with participants trying to figure out and 'live' the routes. This was especially the case for the simple map created by $\mathrm{P} 1$.

Besides abstract visualisations, objectivity and efficiency were key elements, as illustrated by the following explanations: "clear and concise" (P2-R) and "reduce the subjective” (P3-R). Moreover, just as with the first Data Diary, icons and symbols remain the main tool to encode data (see P1 and P8). Here, the work of P8 stands out, as they combined the basic principles of visualisation to layer different information, such as purpose (e.g. food, friend, school), how they felt during their trip, time taken, and mode of transport (e.g. run or walk). Furthermore, P7's sonification stood out. This represents whether a path was useful (e.g. to grab food), or aimless and for procrastination. Useful paths were represented by lower notes, whereas procrastination was assigned the higher tones. Using a music box, one can play the data representation. The creation of this sonification might have been influenced by a lecture in the prior week about different modalities that could be used to represent data.

\subsection{Data Diary 3: Counting and Categorising}

Our next challenge was getting students to create physicalisations (see figure 3). Participants were asked to count and categorise data from their everyday lives, and represent it using physical objects. This resulted in a clear departure from participants' previous work. Whereas the first two weeks embraced creative visualisation methods, inspired by InfoVis and "Dear Data", participants now had to find new ways to communicate data. For three participants, this resulted in a literal representation, where the objects represent themselves, as can be seen with P2. Noticeably, P2's visual style changed drastically with this assignment, being their first diary that considers aesthetics. The five others created mappings to objects or materials, as can be seen with P4 and P8. Key to this was iteration. For example, P4 started out by mapping the weather to scarves. Their process quickly became more poetic, with a physicalisation made from jewellery boxes representing social group sizes (e.g. the biggest box represents the biggest social group) and the order in which they wanted to send them Christmas greetings. Similarly, P8 started by categorising their vitamins and supplements, but moved to representing the Black Friday emails they had received, where each object represents the type of offer (e.g. nail polish represents a beauty deal), and lines of pebbles to represent internet usage in calls with their partner.

P3's work sits somewhere between being literal and metaphorical, using phone chargers and cables to represent the number and length of phone calls for a number of people. Here they did a literal 

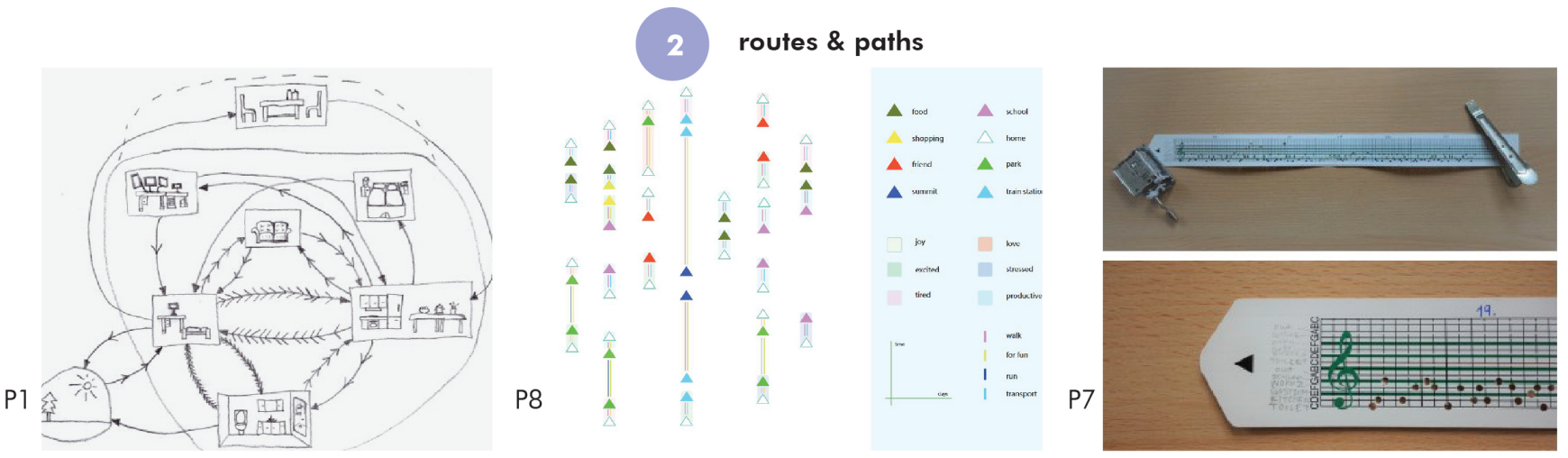

Figure 2: Three exemplary outcomes for the second Data Diary assignment. Left: P1's map shows the routes taken in their home (and outside) and their frequency, resulting in an easily legible representation. The icons represent the locations and the arrows indicate the routes. The frequency of each route is indicated by the number of arrowheads on each arrow. Middle: P8's representation requires a legend and results in an abstract pattern of the routes they travelled outside (it encodes goals/destination = triangle colour, time taken = line length, mode of transportation and mood = colour(s) of line). Right: P7's sonification represents the routes between rooms at home, with different encodings for purposeful and aimless movement.

3 Counting \& categorising
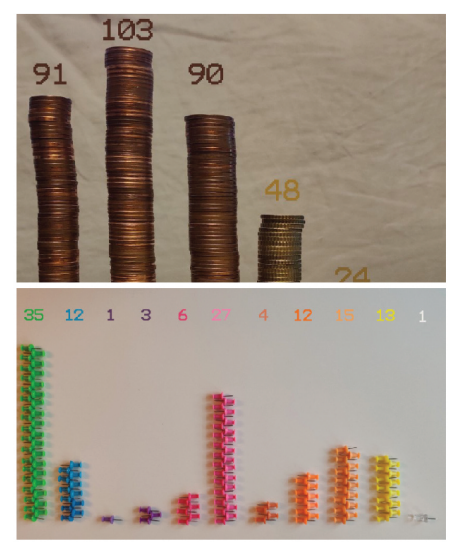

P2

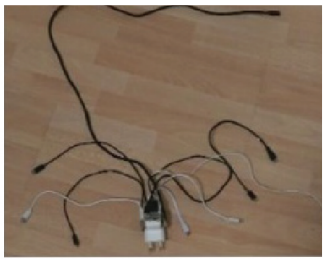

P3
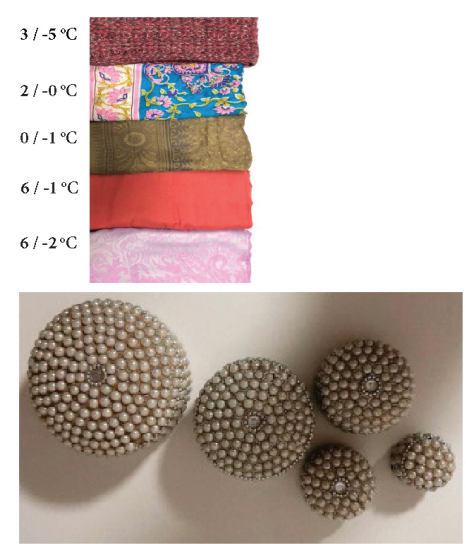

P4

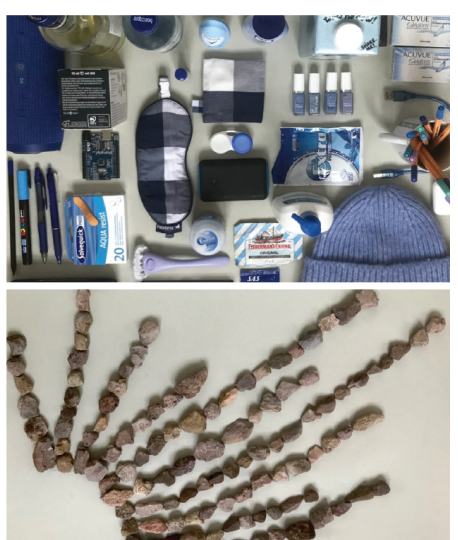

P8

Literal representation

Metaphorical representations

Figure 3: Four Data Diaries created for the third assignment. Having to physicalise their data resulted in literal and metaphorical representations, and one in-between. Left: A literal representation by P2, who counted and categorised various objects (here: colour categorised pins and value-sorted coins). Middle left: P3's physicalisation has both literal and metaphorical qualities. They used phone chargers and cables to represent the number of phone calls (number of cables) and length of each call (cable length) with a person. Middle right: P4 metaphorically represented the weather with coloured scarves and used jewellery boxes to represent the size of social groups (e.g. family, friends) and the order they would receive Christmas greetings in. Right: P8's metaphorical representations - different objects represent Black-Friday deals received per email and a pebble arrangement represents the "amount of Internet used in a call with my partner".

counting, but used the material metaphorically; Giving an interesting visual aesthetic.

During this assignment, participants also started to explore more subjective topics. For example, P6 represented their dreams, P4 their workload, and P8 their emotions.
Lastly, this Data Diary marks the start of two important themes that ran throughout the project: materiality and habits. Due to the physicality of the objects, participants started to think about and work with materiality. This would later become one of the most important elements (see section 5.3). Moreover, here participants 

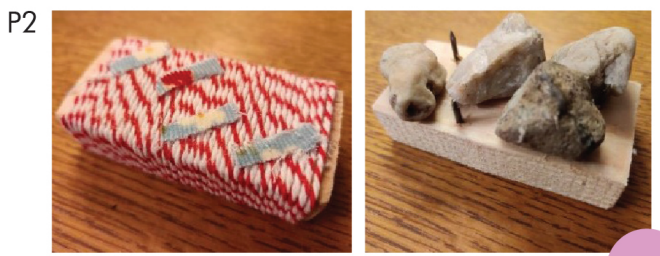

P7

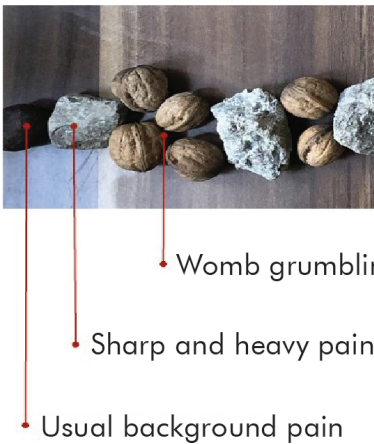

P3

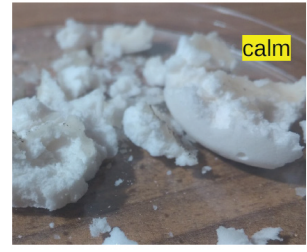

Senses / sensations

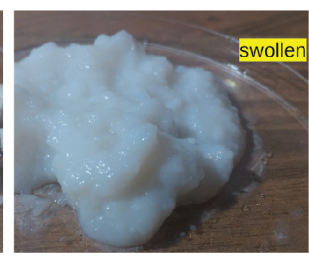

swollen

4

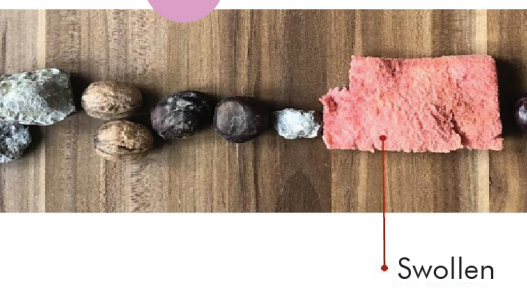

(1)

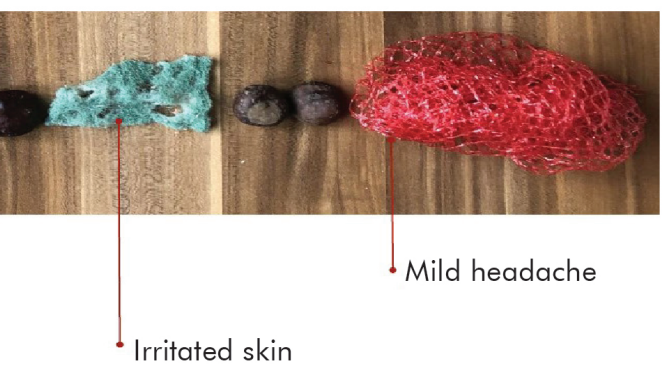

Figure 4: Three physicalisations which focus on the tactile modality. Top left: The feelboxes created by P2 represent music. Top right: P3's tapioca textures represent bodily feelings, such as 'swollen' as well as emotional feelings, such as 'calm'. P3 transformed the tapioca from one stage to the next, so the previous representation would be gone. Bottom: A timeline of P7's menstrual pain.

begin to explore and represent their habits (P5 and P7), which continued to be a popular topic.

\subsection{Data Diary 4: Senses and Sensations}

For the fourth Data Diary (see figure 4), participants had to create a haptification of the senses (sensual perceptions) or sensations they experienced. This challenged participants to explore and think about the subjective elements of data, as stated by P3: "I had to deal with subjective data". The combination of topic and modality resulted in representations of personal topics -such as menstrual pain (P7)-, using personal mappings between data and material.

Similar to the previous Data Diary, this one breaks with the representations created in earlier assignments. Most notably, the view from afar disappears. With this, we mean that the ability to look at the data representation and retrieve the information by looking at it without having to explore its 3D-nature, disappears. Instead, the 3D-nature, material characteristics, and even their temporality (P3), play a crucial role in communicating the data. Lastly, we noted that most of the physicalisations focus on using one material (e.g. P3). Only P2 and P7 used multiple materials for their representation. The materials used by themselves were a wooden stick, tapioca, paper, sand, and tape. The latter three materials provide almost endless affordances, or possibilities to be shaped and manipulated.

\subsection{Data Diary 5: How I spent my time}

The last Data Diary required participants to represent how they spent their time, using movement. Our initial thought behind this assignment was that students would build or create something which moves. However, this modality proved to be the most difficult, with some participants (P1, P3, and P8) deciding to use something else, or not use it at all and focus on a static representation (P1), or use movement, but not for communicating data (P8). From the three participants who did use movement to communicate data (see Figure 5), two used the human body, moving away from what is normally considered a physicalisation. A reason for this could be that the body does not require technical knowledge to create movement: "I don't know anything about how to create mechanisms, like moving stuff" (P4).

Similar to the previous two assignments, participants mostly used personal or subjective mappings and symbolism to represent data. This can be seen in the abstract animation created by P6 and the movements generated by $\mathrm{P} 4$. P4 mapped their activities to different styles of walking and running, with styles they enjoyed doing representing enjoyable tasks, and vice versa. $\mathrm{P7}$ on the other hand, used a mix of made-up movements and mimicry, such as typing and scrolling. The represented activities all used hands, to ensure that data and representation align: P7: "if I decided that I am going to use hands and in the end I do so many things where it is super difficult to represent by hands, then the whole thing, for me, is just kind of meaningless".

Next to this, the materiality of data remained an important element. For example, instead of using movement to convey data, P3 decided to continue exploring haptics. This marked the third week of continued fascination with materiality for P3. However, in this week, P3 discovered what, according to them, are the limits of materiality, namely its subjectivity: "I was not able to find a "natural" link between activities and surfaces and were only able to vaguely map them" (P3-R). This resulted in a shift with their final prototype, as we will discuss next. 


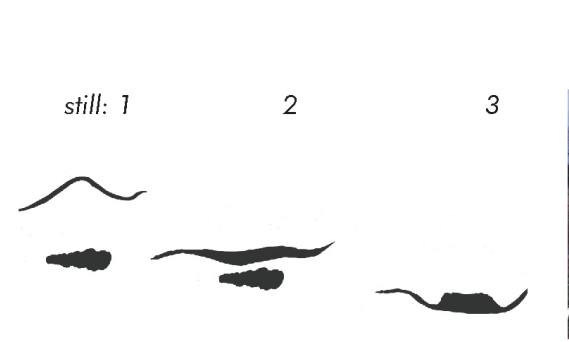

P6

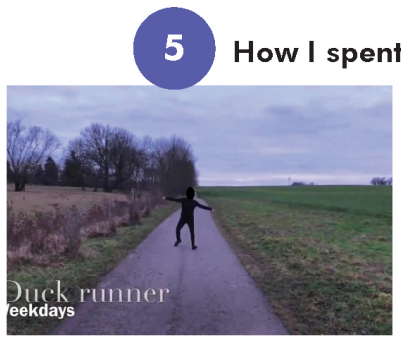

P4

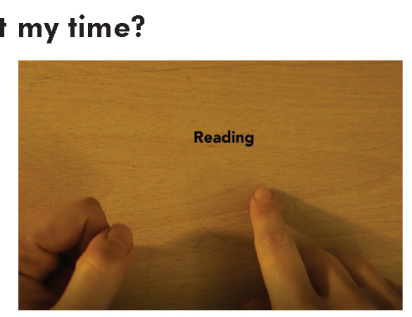

P7

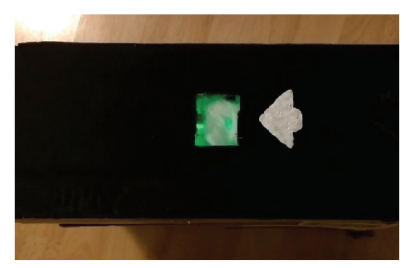

P3

Figure 5: For the fifth Data Diary participants were asked to use movement to communicate data. Left: P6's animation video represents the activities of one day, such as eating, dreaming, gaming, to buying a present. Middle left: $P 4$ used different types of running to represent their activities throughout the week. Each style of running represents a different activity, with the quality of the run representing the qualitative aspects of the activity. For example whether it is enjoyable or hectic. Middle right: P7 used hand gestures and movement patterns to represent activities they had performed with their hands (some abstract, some mimicking actions such as scrolling on their phone). Right: P3 continued to explore materiality and created an interactive box, where activities are represented by different materials. A motor moves the different materials into the touch area (left of the arrow).

\subsection{Final Prototypes}

For the final assignment, participants had to create an interactive physicalisation which represents personal data. The data source was theirs to pick, which resulted in three groups: first, some participants (P1 and P5) picked an interest they discovered during the class. Second, some participants (P2, P3, P4, P5 and P6) chose a topic relevant to their personal or social lives. Third, some participants (P7 and P8) decided to focus on how data can force change. Furthermore, the physicalisations were created for different purposes, with two participants (P1 and P7) creating something that contributes to data activism [55], five making a physicalisation which offers personal insights (P2, P4, P5, P6 and P8), and one wanting to communicate to someone else (P3). Despite the variety of physicalisations, emotion (P5 and P6) and calendar data (P2, P3, and P7) were the most commonly used. A reason for this could be that these are data sources which you already track or are at least aware of. Figure 6 and 7 show and explain the created physicalisations.

Looking at these final creations, we see that some of the participants reverted in their aims and styles, compared to the development that had taken place over the Data Diaries. Firstly, we can see that the 'view from afar' is back, with all physicalisations allowing one to purely visually retrieve the data, without having to explore the artefact's 3D-nature. Note that we do not mean that the 3D-nature does not play a role. For example, in P1's design, it plays a crucial role in highlighting the data plurality and providing an engaging interaction with the data: creating suspense, hiding some of the data until you pull the arrow out. The design aims to make aware of the full story, by having users actively interact with the physicalisation. Concerning P3's journey, we see that they now evaded the topic of materiality, as they were not able to find a way to objectively link materials and data. Instead, they explored how the shape of an object can contribute to the story: "Then it started not to really be about the material, but more like the story you can tell with the form and with an object, and in the end decided to tell my story with this metaphor of the bird" (P3). Besides these changes in direction (or reversals), new elements emerged. A key aspect is the return of 'romantic' ideas. Instead of focusing on the objective, participants decided to track emotions, and represented them through nature and metaphors. Noteworthy, both physicalisations that represent emotions, use nature to represent it: P5 used a mouth and P6 made a heart from moss.

Finally, as participants had to create an interactive physicalisation, movement played an important role. Movement was either used to communicate data (e.g. P7's Poking Finger), transition between states (P3), or enforce active participation. The latter was the case for P1, where the user had to pull out the data to discover the full story. Other modalities used for the interactive prototypes were sound (P2's marble run) and time (P7's moss heart).

\section{OBSERVATIONS AND INSIGHTS}

From the analysis of the interviews, reports, and Data Diaries we found that participants have a different understanding of visualisations and physicalisations. Data physicalisations are considered to be lively: "a conversation starter and a friend" (P8-R), which evoke more feelings than visualisations: "that can make you feel a bit more [...] when you see like a flower dying that represents your data, instead just a graph" (P6). On the other hand, compared to visualisations, physicalisations are seen as "abstract" (P4, P6, and P7) and not as easy as just drawing your data (P6 and P7), using pen and paper (P3, P6, and P7). However, the main difference between visualising and physicalising data is the experienced freedom. Instead of having to follow the rules of visualisation (e.g. Bertin's visual variables [4]), participants felt they could use anything to physicalise their data. This meant that participants had to find new ways of encoding data, which often relied on personal mappings and reduction. Despite all differences, essential to all data representations is that they convey meaning.

In learning to create physicalisations, participants went through four stages in their thinking and the challenges encountered. This started with feeling overwhelmed, and ended with creating a symbiosis between the story to tell, data, method, and material. Via the Data Diaries, participants progressed through these stages, and 


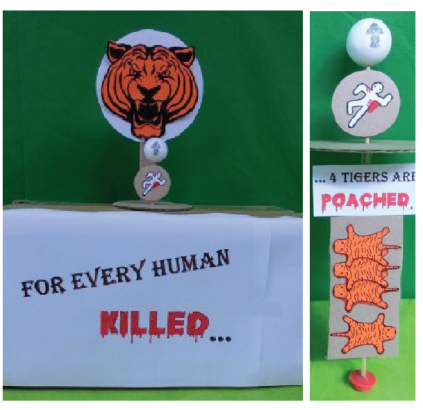

P1: Tigers versus Humans

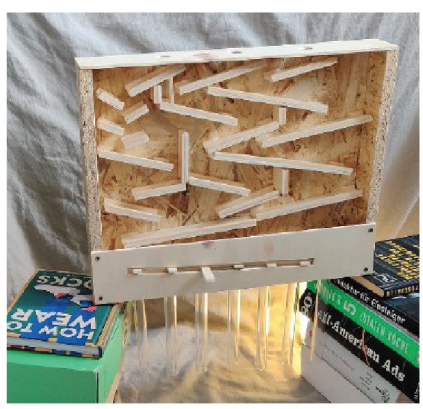

P2: "The What Are You Even Doing? - Run"

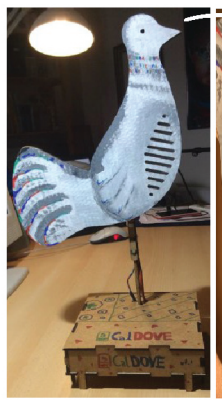

P3: CallDove

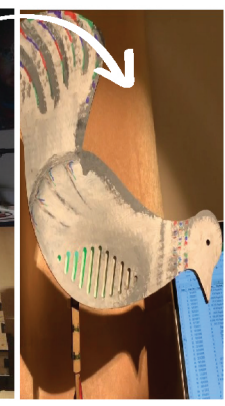

GOODBYE GODBYE

P4: Hellos and Goodbyes

Figure 6: Interactive physicalisations by P1, P2, P3, and P4. Left: P1 explored how a physicalisation could highlight the full story behind data, as most data representations focus on one side of a story (in this case, the fatalities caused by tigers). At first, you only see a looming tiger above a dead human. On closer inspection, the artefact invites you to 'lift' the data by pulling the arrow out. By doing so, one discovers that for each human killed by a tiger, four tigers are poached by humans. Middle left: P2 created a marble run for time management, that represents tasks to complete and completed tasks. One can pick from four colours of marbles: one represents a finished task, the others display different levels of urgency and importance. One then picks one of three routes, whose length indicates urgency (short runs are urgent) and the loudness of a run indicates importance (loud is important). The marbles end up in one of five tubes that represent weekdays. Middle right: P3 designed a physicalisation which communicates their schedule to their (remote) father as an awareness display. This would connect them during P3's busy periods. The colour of the lights in the dove's breast indicate the appointment category and the rotational degree with which the dove tilts indicates the workload of the day. The transitions between appointments are indicated by movement and sound, as the dove rotates its head (making noise). Right: P4 returned to the first Data Diary and represented the number of hellos and goodbyes. Hellos are indicated by the 'LL' moving up and down. A long move or travel indicates a high number of hellos. Similarly, the number of goodbyes is indicated by the rotational speed of the 'OO'.

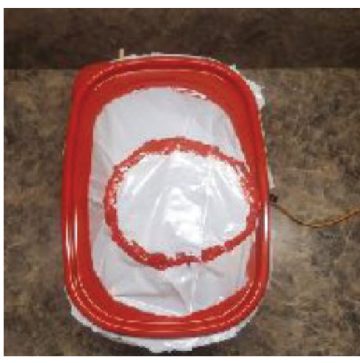

P5: Emotions represented

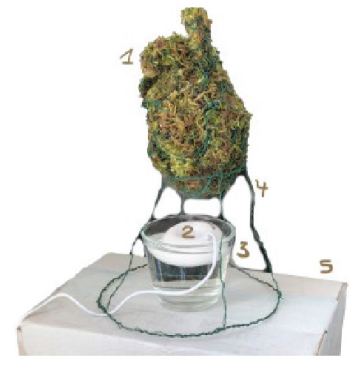

P6: Moss Heart

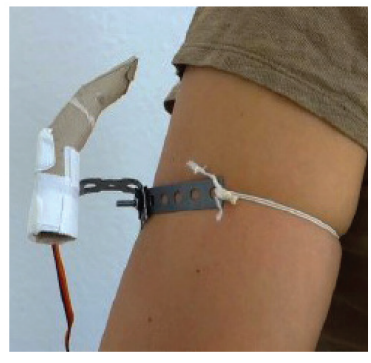

P7: Poking Finger

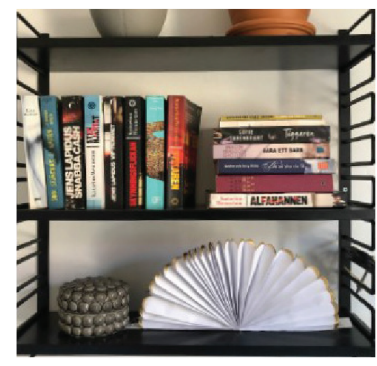

P8: Energy consumption

Figure 7: The interactive physicalisations created by P5, P6, P7, and P8. Left: P5 tried to create a mouth which represents the user's emotional state. Middle left: $\mathbf{P 6}$ created a moss heart which represents your emotional state. In an app, the user indicates how they feel that day. If they feel good, the moss heart receives water via a humidifier, but if they feel unhappy, it remains dry. The physicalisation thus is a literal representation of how your heart 'feels', with positive feelings resulting in a green and lively moss heart and negative feelings in a brown heart. Middle right: P7's Poking Finger: "a satirical data-object, aims to make people aware of the omnipresent social control technologies". This is a wearable to be worn on the upper arm and connected to the phone. It will tap the user three times to indicate calendar notifications for activities and appointments, continually tap until an incoming call is answered, and poke when the calendar indicates a new work task. One could also connect to somebody else's calendar, thereby experiencing other people's daily rhythm of busyness. Right: P8's physicalisation shows your energy consumption. When fully folded out, it emits light (like a lamp), indicating your energy consumption is low. As the user consumes energy, the device slowly folds and the light dims, until fully closed and dimmed.

discovered that physicalisations blur the line between the qualitative and quantitative. Therefore, in order to design physicalisations, they needed to change their notion (understanding) of data.
In the following, we discuss these findings: 1) Freedom of Physicalisation, 2) Data Representations Should Convey Meaning, 3) Materiality of Data, and 4) the Effect of the Data Diaries. As four 
Table 2: The three different levels of reduction.

\begin{tabular}{lll}
\hline To tell a clear story & Limit possibilities & Reduce complexity \\
\hline Reduction of number of data entries & Reductions of amount of options & Categorising data \\
P7-R: "Sometimes I exclude certain data en- & P3-R: "I struggled to find any convincing ap- & P2-R: "I picked songs as the dataset and break \\
tries merely because too much of them would & proach for my final project. So I aimed to de- & those down in categories which would in turn \\
obscure a legible narrative. [...] In order to & cide on more restrictions." & be mapped to materials and textures." \\
create a clean and strong narrative, I filter out & & \\
many repetitive data and present only those I & & \\
grant as "representative"." & & \\
\hline
\end{tabular}

participants ( $\mathrm{P} 3, \mathrm{P} 4, \mathrm{P} 6$, and $\mathrm{P} 7)$ agreed to take part in the interview, their quotes and insights are more commonly referred to than those of others, as clarification on their written and created work could be asked. A birds-eye view of the important discoveries, changes, and challenges throughout the Data Diaries and final prototype can found in the Appendix B.

\subsection{The Freedom of Physicalisation}

As the Data Diaries moved from visualisation to physicalisation, participants learned about their differences by creating them. Visualisations were seen as something you do with pen and paper, which made them easier to create: $\mathrm{P} 7$ on physicalisation: "It is not as easy as 'yeah, I can just draw it on paper"' and P6: "I mean drawing is always easier because yeah you can just draw what you think". Moreover, visualisation demands that you follow certain rules: "we used paper and pen and it has some rules and yeah..." (P4). These rules make you approach visualisation with a fixed mindset: "Ijust needed to tick off all these checkboxes" (P3 on the first Data Diary), and if they are not followed correctly, it results in a 'mess', as stressed by P4: "My first task, it was too much I would say, mess. I was using different shapes, different colours and all."

Compared to this, physicalisation does not limit you, as P3 expressed: "With the physicalisation you are not only allowed to use pen and paper, but everything that surrounds you [...]. And somehow every object could be part of your physicalisation and there you get such a degree of freedom that it is much less like an algorithm you follow and more like being an artist". Because of this freedom, participants stated that every material could be used for a physicalisation: "you don't have to rely on material, [you] can just basically take anything you want and it will work out" (P6).

As the rules of visualisation no longer applied, participants had to find new ways of communicating and dealing with data, this resulted in new mapping approaches and the need for reduction. We discuss these themes below:

5.1.1 Mapping: Icons, Indexes, Metaphors, and Personal Mappings. While participants could use icons to capture data in visualisations (as can be seen in the first two Data Diaries), this option disappeared for the physicalisations. Here, participants had to find new ways of mapping data that would allow them to communicate the information, which was not an easy challenge. Throughout the Data Diaries, we can see that participants found various ways of coping, the most common being the use of metaphors and symbolism, and personal mappings. The former can be seen in the final prototype of P3, where a pigeon-shaped data physicalisation communicates your personal schedule to someone else, referencing to how pigeons were used for distance communication, and P4's fourth Data Diary where: "if the load is more, it showed by more amount of sand and vice versa" ( $\mathrm{P} 4-\mathrm{R})$.

Regarding personal mappings, participants created new mappings to explain data. Examples of personal mappings include P8's fifth Data Diary, where they used coffee and milk to explain their estimated screen time versus the actual amount: "The black coffee is my guess, and the milk is the gap to reality" (P8-R), or P4's fifth Data Dairy, where they created different types of running to communicate how they spent their time.

5.1.2 Reduction. Compared to visualisations, where participants could represent all the data points they encountered: "With visualisation I can really represent things that is detailed (sic.), so I am just going to write everything down about this one single point" (P7), the creation of physicalisations requires reduction [61]. This reduction could happen on three levels, each with its own rationale. The levels and reasons, together with a descriptive quote can be seen in Table 2 .

\subsection{Data Representations Should Convey Meaning}

Despite the differences between physicalisation and visualisation, participants believed that data representations should have a purpose: "The aim of visualization and physicalizing of data is to enhance the understanding of data" (P4-R). Therefore, physicalisations should tell us their meaning: "whatever we were designing, it tells us what it means" (P4), something which was not always easy: "It is also difficult to understand the object on its own" (P2-R). The feeling that the object should explain itself, was further defined by P7, who made the distinction between personal data physicalisations and physicalisations that explain data to others: "I feel there is a goal of data representation is that I want to say something through this [object], that is evidence. So represent that in a way that not just me, but everyone can understand why, what is going on, and how this data is being used in this way. But if it is just for myself, I can do like super abstract things".

Although both visualisations and physicalisations should convey meaning, physicalisations were seen as a human way of communicating data: Interviewer: What did you learn about data from creating data physicalisations? P3: "data is the information for computers [...] if we really want to make the data accessible for humans, 
we need to find a different form, because the form the computer needs is not suitable anymore."

\subsection{Materiality of Data}

From the third Data Diary (counting and categorising) on, participants were asked to create physical data representations. From this moment on, one of their main challenges was the materiality of physicalisations. Here, they had to think of (and often struggled with) finding a harmony between data source and material. For instance, P2 described in their report: "Choosing the right data set and find (sic.) a fitting object for that set turned out to be a much more challenging task than initially thought" (P2-R). Also, it was difficult to let the material do the talking, as explained by P3: "I was not able to find a "natural" link between activities and surfaces and were (sic.) only able to vaguely map them because of their characteristic qualities" (P3-R). However, throughout the Data Dairies, we see that participants became more skilled and less taken aback by the idea of physicalising data. Reflecting upon their learning journey, we identify four stages:

Initially, at the first stage, participants feel overwhelmed by the idea of representing data through material artefacts: "Physicalising data is a very abstract idea [...]. How does one go about transferring numbers and words into an object of the real word?” (P2-R). In the second stage, participants overcome these initial concerns, utilising their experience with visualisations. They search for materials and constellations that can universally explain the data to everyone, which turned out to be impossible: "I was not able to find a "natural" link between activities and surfaces and were [was] only able to vaguely map them because of their characteristic qualities (sic.)" (P3$\mathrm{R})$. This realisation brings participants to the third stage, where they move away from the idea of finding materials that can universally explain data. Instead, they embrace the freedom of physicalisation and realise that everything may be utilised in a physicalisation. P4: "sometimes I was thinking: we should select some wood objects, we should select something, it should not be anything. But now I can see that we can use, you can use everything". In the fourth and final stage, this changes again. Participants realise that there has to be a link between the material and the story you want to tell. P7 told us: "at the beginning of the course, I would think about the material that would represent like roughly this data. But in the end, the materiality would decide [...] what aspects of this data is represented." In this stage, all elements of the physicalisation process (such as method of collection, cleaning the data set, material, etc.) come together, in order to tell the story of the data. P7 continues: "The most important thing is that this data needs to deliver a message [...], when I think about how I am going to represent it and then I start to collect this." A visual overview of the four stages can be seen in Figure 8.

5.3.1 Quantitative versus Qualitative. Another challenge was finding ways of 'quantifying' personal data. While this was easier for assignments which already suggested enumerable categories, such as the number of hellos and goodbyes / compliments and apologies in Data Dairy 1 or counting and categorising objects in Data Diary 3 , it was more difficult for topics which do not yet exist numerically, such as Data Diary 4: senses and sensations. The following quote of $\mathrm{P} 2$ serves as example: (P2-R on their final prototype): "How do you quantify productivity?". [...] After all, productivity is always a personal estimation too".

Whereas participants were initially concerned with quantitative values of data: "I never take a data point and think about what feature it has, besides [...] value" (P7), the Data Diaries highlighted the qualitative aspects of data. As each Data Diary resulted in a variety of approaches in what was counted and how (just hello's and goodbyes, or also the mode of communication, feelings experienced, etc.), the decisions involved in deciding which variables to track and how to categorise these became visible. Making participants experience that often data categories are not clear-cut.

The tension between quantitative and qualitative further increased when creating physicalisations. This can be seen in the third Data Diary, where we have the first introduction of personal mappings (e.g. P4's scarves that represent weather) and topics which become more subjective (P6 represents their dreams). With physicalisations, the data could not be solely numerical as the material nature enforces a link to the data's origin. P7 described: "Physicalisation, it has more to do with the materials that I use, to represent a kind of. Goes back to the thing which it was". A similar point is made by Offenhuber [47]. Because of the link to the origin, not only the way of representing matters, but also how data is collected - P7 explains what they learned about data from physicalising it: "How to present it, its relation to how to collect it". As such not only the quantitative aspects were considered, but the qualitative as well, as exemplified by P3: "It helped me to decide to more focus on the perception of this [...] physicalisation. And maybe how to encode it more with also the feelings of the people that are way more uncertain and way less objective and way less well defined."

As participants became aware of the qualitative nature of data and the role of qualitative aspects (e.g. encoding the uncertainty of the data into the representation), they realised that subjective topics can also be seen and used as data sources. This expanded their perception of what data can be: "Feelings can be also encoded too, so I think that this evolved my perceptions on the limitations for data" (P3).

\subsection{The Effect of the Data Diaries}

The last themes we discuss concern the effect that the Data Diaries had on participants. We highlight the Didactic Effect of the Data Diaries and the Notion of Data.

5.4.1 Didactic Effect of the Data Diaries. As our project took place online, one of the challenges was to keep participants engaged and create an atmosphere in which they would feel safe sharing personal data. This seems to have been accomplished through the Data Diaries, which facilitated an open environment: "I liked the ability to experiment and express ourselves quite openly" (P5-R).

Moreover, the Data Diaries had didactic effects. They helped participants gain an understanding of the variety of data sources they could use and helped them realise what they wanted to create: "My Data Diaries have made me realize that I wanted to create something - at least partly - analog" (P6-R). Furthermore, they helped participants understand the process of collecting and representing data, as highlighted by the following quotes from P2: "During the second week, data on paths and routes was collected. This time with more of an idea behind it and more data dimensions, the visualisation 


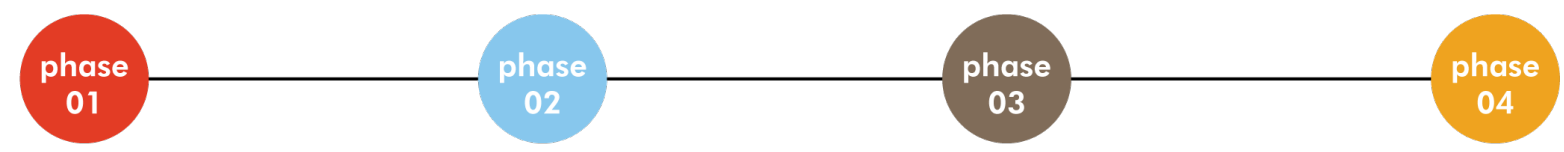

people feel overwhelmed by the idea of physicalising data people apply visualisation principles to physicalisations everything can be used for the creation of physicalisations people select materials which contribute to the story of the physicalisation

Figure 8: The four learning stages of materiality.

is already much more clear and concise." and: "The Data Diaries were a great help to understand the different aspects of what it takes to create a data physicalisation" (P2-R). Lastly, the Data Diaries primed participants to observe the data around them: P4 on the third Data Diary: "during that time, I was not able to observe everything. But after this Data Diary, I am observing everything”. All together, these didactic effects eased the teaching of physicalisation, as participants iteratively learned about the data around them, and acquired the skills to select a data source and represent it.

Key to the these effects was that participants had to collect and represent data themselves, as explained by the following two quotes of P6: "Normally you just have the statistics [...] and now you just have the raw data and you have to reflect on it in order to know how to represent it and you can think more about what the data actually means to you" and: "That you have to actually create the physicalisation on your own and not just have something given to you [...], that you actually have to work on it on your own and think of an object that fits the data".

Finally, as participants had to track elements from their daily lives, they noticed and commented on having a desire to or to consciously influence their data, due to their awareness of it being documented: "What if I do not go out one day, so the data collection might be zero on that day" (P4-R) and: "If I know I am going to collect this data, I tend to do something that makes this data look like such" (P7). The latter was further explained, when P7 talked about their sonification in Data Diary 2: "When I was punching those holes, I was thinking: 'yeah, I am not going to the toilet so much, because it is going to sound weird'." This could have helped participants realise that data is not always as neutral as commonly believed: "There is a human behind every function that encodes the data, so it's always subjective and not only objective like you would expect for the data to $b e ”(\mathrm{P} 3)$.

5.4.2 The Notion of Data. As highlighted above, participants started to realise that data is not neutral and objective. This ties into our main observation; our analysis indicates that physicalising data both demands and facilitates a change in how we see and think about data. At the start of the project, participants thought of data as "just boring numbers and statistics" (P6), "abstract" (P1, P2, P3, $\mathrm{P} 4)$, "very objective and very efficient" (P3), "information that is encoded for the computer" (P3), and a way to "reduce everything into some point" as such making it "concrete" (P7).

However, throughout the Data Diaries, we can see a shift in this notion. With the first two Data Diaries, participants still had their old understanding of data. In the follow-up interview P6 reflected: "with the first one you still have the old thinking of data how just represents like numbers". This can also be seen in some of the analytical approaches and abstractions used for the visualisations, as explained by P3: "In my first Data Diaries I tried to be very objective and formal, in the second data representation I also tried to really achieve a representation that is completely free from those subjective algorithms, or decisions you could make, which is only performing very mathematical, very general transformation that could also apply to other forms of data, to abstract the data to a point where it can't be reconstructed".

This continued until the third assignment, where participants had to create their first physicalisation. At this point, physicalising data was still an abstract idea to them: "Physicalising data is a very abstract idea, since data is made up of numbers and multiple dimensions a lot of the time. So how does one go about transferring numbers and words into an object of the real world?" (P2-R). This perspective was heavily influenced by participants' experience with visualisations, as P3 expressed: "My expectations on physicalisations were different before, because I thought more, like they have to be efficient and they need to give me all those answers that I want to know". This understanding can for example be seen in the third Data Dairy created by P5, which focused on creating an efficient (and useful) representation: "I had only recently moved into my new apartment and was still trying to organize my kitchen goods efficiently, I thought I would track the general pattern of use" (P5-R) and P2's third Data Diary, which focused on a rather literal categorisation of data and represented it using aesthetics common to visualisation, as can be seen in Figure 3 (colour-sorting and bar charts).

However, we can also see a shift in how participants approached representing data in this assignment: representations become more poetic, topics more abstract and subjective. This is evident in the work of P4 (see Figure 3 and 5). With physicalisations, participants needed to re-explore how to represent data: "According to me, $2 D$ was easier than $3 D$ since it reminded more of the classical ways of presenting data and when doing something in $3 D$, I had to rethink the meaning of things" (P8-R). This had them realise that their old understanding of data representations did not hold up anymore. P3 explained: "I didn't struggle with that one [Data Diary 1] and that changed with the next Data Diaries [Data Diary 3 till 5]. There I really felt the struggle and I think this was also the point where I needed to change my habits".

The friction with participants' initial notion of data continued with the fourth Data Diary, where they had to track the "subjective topic" (P3) 'Senses and Sensations'. This restricted any exact logging of data, which resulted in a different representation approach: "I cannot say: 'okay, this is the point of my pain from this time to that' 

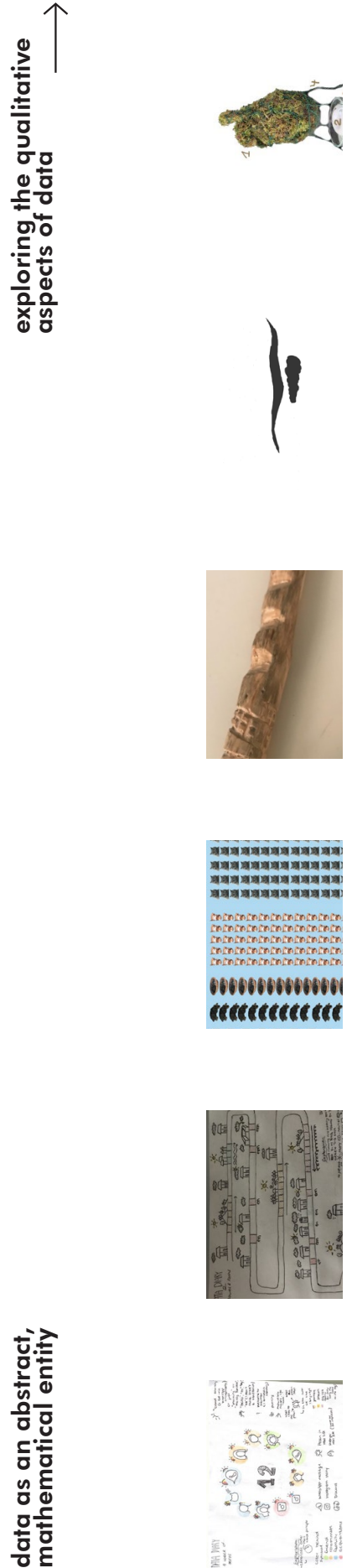
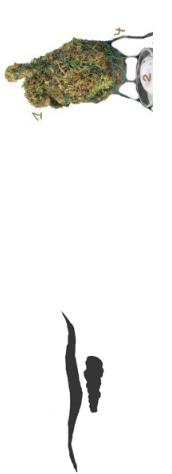

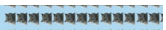

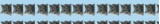
19

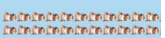

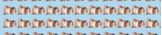

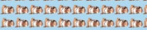

0000000060600

entectetetert

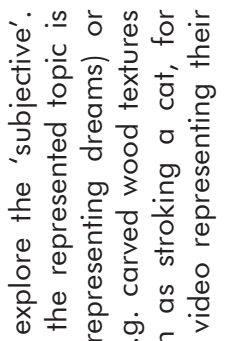

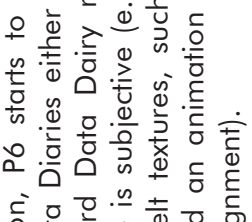

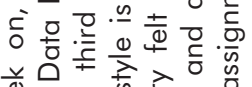

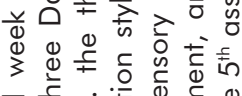

은 $\dot{0}$ 舟

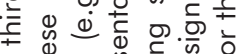

(

千 ס

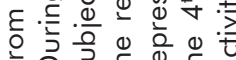
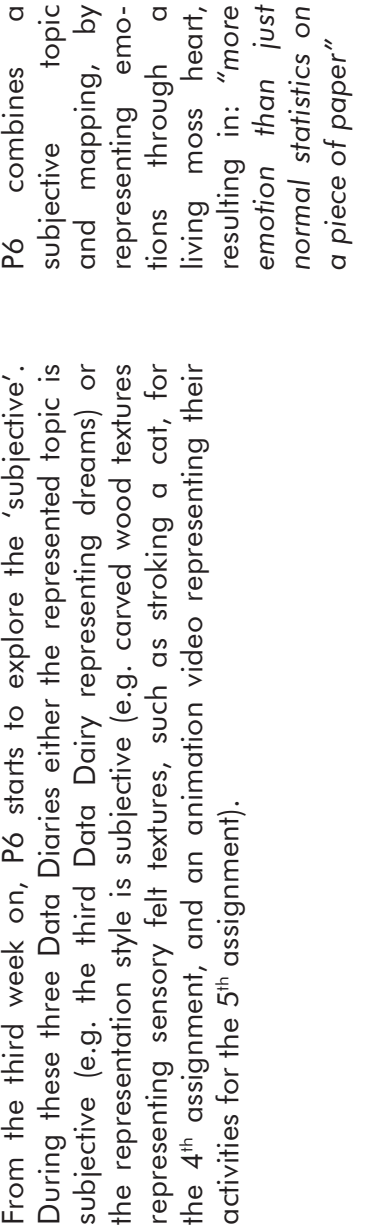

$\frac{\searrow}{20}$
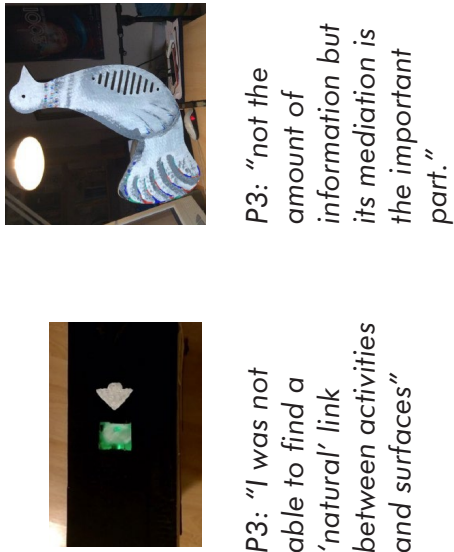

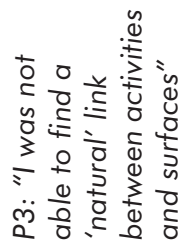
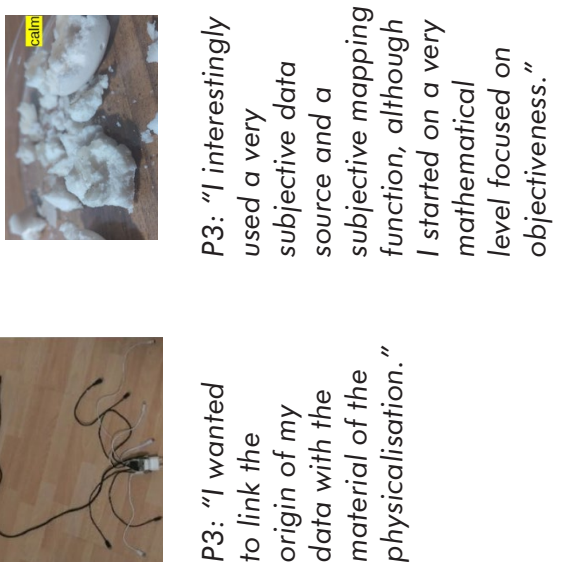
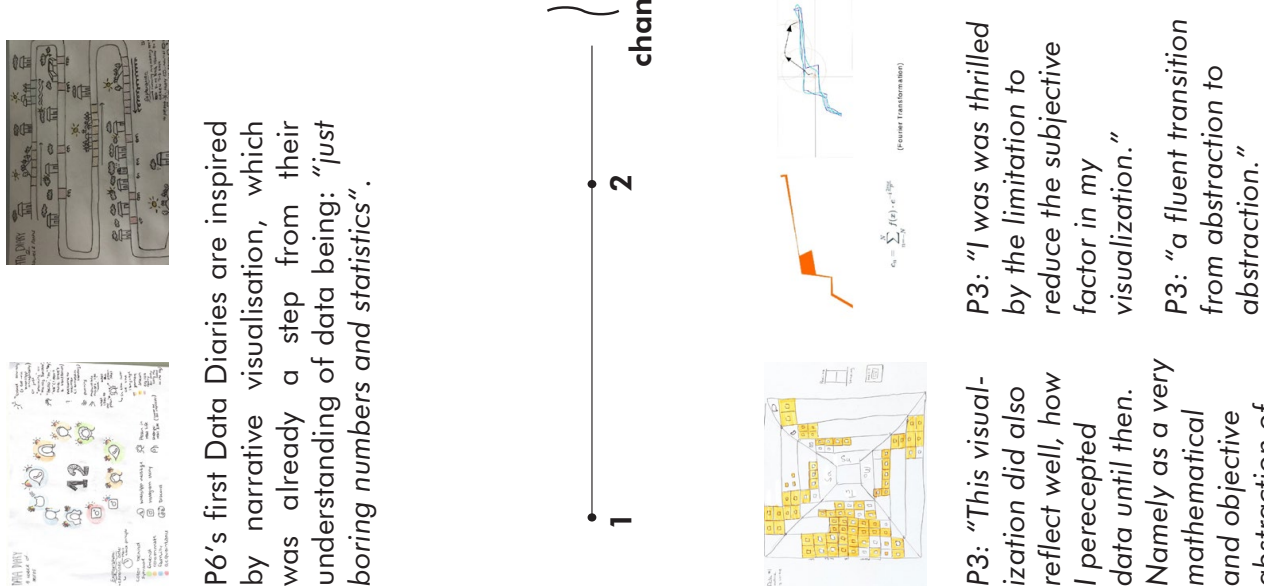

ใิ

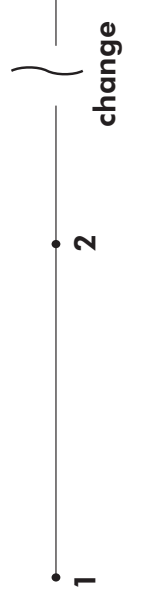

Figure 9: A full-page overview visualisation exemplifying the change in student's notion of data throughout the assignments. Due to the length of the figure description, the full description can be found in Appendix $C$. The brief description is as follows: a figure consisting of a line with at the top, P6's Data Diaries, final prototype, and our observations. Below the line, we have the same for P3, only with quotes from P3 instead of our observations. The quotes and observations show that at the start of the project, students saw data as mathematical, and "just boring numbers". Through the assignments, they started to embrace subjective topics (e.g. dreams) and mappings (e.g. textures they felt made sense to express sensations such as calm). With their final prototype, the students expressed that physicalisation focus on the mediation of data (P3) and are more emotional than statistics on a piece of paper (P6). 
and how that measures. So everything is kind of like [...] a reflection" (P7).

The physicalisations challenged participants to rethink what they knew about data representations: "I never questioned that digital screens were the main way to represent data [...] and therefore I never really thought about the potential that physical objects have in conveying data. That this concept was new to me can to a certain extent be seen in my data diaries (especially 3 and 5), as looking back, they are not nearly as imaginative in utilizing the strengths of the physical realm as they could have been" (P1-R). Moreover, it made participants realise there are other ways of communicating data than those commonly used: "A physicalisation that is not aiming to provide many (sic.) information and give a lot of answers, but just is some kind of link or little window where you can maybe peep with your eye through and then you can take a look into a life of a different person and this is not about efficiency anymore, so I really needed that change" (P3). As highlighted by this quote, participants had to change their notion of data in order to design data physicalisations which are not merely a replica of standard 2D-InfoVis representations.

Whereas participants first saw data as neutral, abstract, and objective -and therefore, data representations should be the samephysicalisations broadened this notion. Participants stated that physicalisations do not necessarily rely on these characteristics (objectivity, neutrality, and efficiency): "It is not really about accuracy, more about: you see this data and you use it to say something that you want to say. Rather than: 'well, what I am presenting here is neutral' - Because it is not" (P7) and "you don't always have to be very precise [...] it does not always have to be so objective and efficient orientated (sic.). That it is somehow [...] the goal to give some kind of emotion or give some kind of meaning to the data" (P3). Instead, physicalisations allow you to focus on how the user perceives the data. P3 wrote: "I need to design my data physicalisation not for a power user like me, but for someone that needs less information. Where not the amount of information but its mediation is the important part".

As such, the physicalisations and Data Diaries forced participants to re-evaluate their notion of data, which was often initially influenced from the use of graphs at school: "Before, I saw it from a school perspective where you have your graphs" (P6). Their idea of what data IS evolved, as shown in answers to the question of what they had learned about data: "I learned that data can be much more than numbers and graphs" (P8-R), "Data isn't just a linear thing, that just boring numbers and statistics and that it can be a lot more" (P6), and "I count everything as data now" (P4). Examples of the evolution in participants' notion of data can be seen in Figure 9, which discusses P6's and P3's progress and moments of change. The works of $\mathrm{P} 3$ are annotated with quotes from their report which explain their notion of data.

\section{DISCUSSION}

The aim of the course that we reflect upon here, was to explore how to teach students - most of whom did not yet have an understanding of working with physical materials - to create data physicalisations based on personal, everyday data. In order to do so, we developed the Data Diaries, which aimed to prepare students for how to create physicalisations based on personal data, and to (instead of posing one big challenge at once) stagger small, manageable challenges that each introduce a new thought or aim. Although the Data Diaries are only one of various possible methods to teach people about the creation of physicalisations, they seem to have been successful in sensitising our participants to many of the issues relevant for the design of physicalisations. It is yet too early to determine the degree to which the Data Diaries support physicalisation literacy, which would necessitate further research. Nonetheless, the Data Diaries have given insights in which elements are supportive for the development of physicalisation literacy, and what hurdles or challenges to prepare for in this process. We now discuss the relation between physicalisations and our notion of data, future work, as well as limitations of our work.

\subsection{Data Diaries and Physicalisation Literacy}

The first element to discuss is materiality. Although the physical nature of physicalisation is considered one of its strengths [28], our work and that of others (e.g. [57]) has indicated that it is also one of the main challenges to overcome. Contrary to visualisations, where participants felt guided by rules and could draw anything using pen and paper, materials are bound to the laws of physics, might already have a meaning of their own, and not be instantly understandable. Furthermore, materials offer numerous new affordances and mapping options, such as texture, temperature, and shape, making it difficult to decide which ones to focus on. To overcome these challenges, our work indicates that people have to move through four learning stages. Where they start or end within these stages will depend and differ per background. For example, design students and artists will likely skip the first stage, being already familiar with working with materials and sensitised to their richness. Nonetheless, it seems important for physicalisation literacy methods to offer space for the gradual development of these stages and to guide students through them. The gradual build-up of the Data Diaries seems to have helped our participants do so.

Secondly, having to track your own data and create your own representations forces you to reflect on your data, its meaning, and how to represent it. By tracking their own data, our participants were forced to make decisions on what to count and how they were going to count it. As such, they became aware of the complexity of data and that not everything can easily be quantified. D'Ignazio has already stated that such an understanding is essential when working with data and teaching (creative) data literacy [12]. Moreover, participants stated that whilst tracking their data they sometimes had a desire to influence it or the way it was going to look, having them change their behaviour (a similar effect is discussed by Lee [36]). These aspects could have helped participants realise that data is not objective, neutral, and easy. Another aspect which could have helped participants realise this was the act of representing their data. Especially with the physicalisations, participants had to find new ways for communicating data. They did so via personal mappings and resorted to reduction of the data, to make their story understandable. This could have heightened awareness that there are always humans behind data and data representations, and that tracking involves many decisions (e.g. what to track and what not to, and how to categorise it). This means data is not 'a given', not objective, but always has subjective elements due to these decisions $[13,17]$. 
These aspects were crucial in shifting our students' notion of physicalisation and data. Therefore, we believe that physicalisation literacy methods benefit from having people track and create their own data physicalisations, and to not solely rely on data sets and physicalisations retrieved from elsewhere. Moreover, focusing on their own data appeared to contribute to a keen motivation in engaging with and thinking about data. This is particularly relevant for an Engineering or Computer Science context, where it is rare for educational classes to discuss or reflect on anything personal. However, learning deepens and engagement intensifies, when it addresses us as individuals [35].

\subsection{Data Physicalisations and Our Notion of Data}

The last element we want to discuss, and probably the most crucial, is the development in participants' understanding, or notion, of data. Although the insight that data physicalisation demands a new perspective on data, is not new (e.g. [47]), the process showcasing the need for designers of physicalisations to change their view, how this changes, and why, is. In reflection and analysis on our taught class, we found that to create physicalisations, our participants needed to change both their understanding of what data representations (should) do and their notion of data. Prior to our project, participants thought of data as abstract, objective, and efficient, and that data representations should embody these aspects. Their understanding aligned with what is commonly thought and taught: that data is digital, immaterial, big, and neutral [10, 13, 17, 24]. However, through the creation of physicalisations, this evolved. Participants realised that physicalisations do not focus on efficiency and representing as much information as possible. Instead, their strength lies with telling a story, allowing the user to see the liveliness of data, and emotionally connect to it ([62]). The stories told with physicalisations do not demand accuracy; they might even benefit from a reduction of information [61]. As physicalisations adhere to their own characteristics, and blur the line between what is traditionally seen as quantitative and qualitative, our notion of data and data representations needs to be broadened. Engaging with this not only develops an understanding of physicalisation, but also expands the understanding of what data representations can do in general. Therefore, we argue that physicalisation literacy methods should guide people towards broadening their notion of data.

Our observations lead us to suggest that one may also utilise this approach to engender thinking about data, and its role and nature. Thus, the creation of physicalisations might even have an educational role as a 'vehicle', e.g. in a philosophy class on data with sociologists or engineers, where the primary aim is not the teaching of visualisation methods, but to foster a critical reflection and discourse of 'what is data'.

\subsection{Future work}

Our work provides insights in which elements are helpful for development of physicalisation literacy. As so far, little is known about physicalisation literacy, we believe it is important to explore what it involves, so that dedicated teaching methods can be developed. Our findings offer insights on directions to further explore with physicalisation teaching methods:
Firstly, even though physicalisations are tangible objects which exist in our world, the created physicalisations mostly remained 'placeless'. Only two participants (P3 and P8) mentioned where their physicalisations would be placed and used. The other designs never discussed this aspect. The absence of location might be due to the fact that data are often immaterial and placeless, somewhere in a cloud [10], and that we taught online (with one student abroad), not being able to talk about locations well-known to all. However, as physicalisations cohabit our world, their location plays a role in how data are conveyed and interpreted [65]. Therefore, it would be interesting to explore how teaching methods can challenge students to think about the location of their physicalisations, and to derive meaning from its embeddedness in a locale [65].

Secondly, we notice that the final physicalisations created in our project mostly focus on the visual. Although physicalisations offer other modes of conveying data, such as haptics, sound, and taste, the visual modality appeared to dominate. This may have occurred because students were well-familiar with traditional visualisations and some even had attended a course on InfoVis. "The privileging of the visual" [39] appears to only have ended in the fourth Data Diary, where participants were required to focus on the tactile modality to communicate data. As physicalisation offers a plethora of opportunities to communicate data via other channels than the visual, it would be interesting for future work to explore how we can guide students to make greater use of these opportunities.

Thirdly, although our work focused on data visualisations and (interactive) physicalisations, the Data Diaries could be used for teaching other types of non-standard representations, such as indexical [48] and autographic representations [46]. Hints of these types can already be seen in the work of our participants (e.g. P3's and P5's fourth Data Diary), without participants knowing or being referred to these concepts. We therefore believe that the Data Diaries are a possibly valuable method for teaching various data representation methods. Future work could explore whether this hypothesis holds up.

Lastly, we used the Data Diaries in an online project. As participants had to present their personal data to us and each other during the online classes, it helped them and us to get to know one another and build team spirit. As the sharing of personal data resulted in a bond, participants felt free to share their struggles, ideas, failed creations, and personal stories. From our experience, such a bond is much more difficult to create in online learning environments (than in traditional face-to-face teaching), where it is tempting to mute the microphone and turn off the camera. Thus, it may be interesting to further explore how the use of personal data can contribute to online teaching.

\subsection{Data Physicalisation and Other Data Artefacts}

As a reflection on the data artefacts presented in this paper, we note that not all align with the definition of physicalisation as stated by Jansen et al. [28]. For example, P7's work Poking Finger could be considered a data sensification [20] and P3's CallDove might rather be considered an ambient display [41]. In both cases, we would argue that the artefacts communicate data and exemplify the limitations of the current working definition of physicalisation [28]. 
Therefore, we consider it fruitful to further explore and discuss the definition of physicalisation, to see whether and how it can encompass a wider range of physical data representations, such as data sensifications [20].

Furthermore, we note overlap between our findings, and the strengths and limitations of Tangible User Interfaces (TUIs). For example, our and previous work indicates that physicalisations benefit from a reduction of information [61], which aligns with the need to remove 'clutter' in TUIs [53, 59]. Moreover, our work shows that physicalisation's tangible nature enforced an acceptance of qualitative data aspects and subjects. Objects have found to be inherently evocative and emotional [58], which would further explain this finding. A last example of overlap is that TUIs have long been used for the education of abstract problems [42, 68]. In our work, participants gained a deeper understanding of the 'abstract' concept data through physicalisations and creating them. Therefore, our findings might not be restricted to physicalisations, but could apply to a broader range of data objects, such as TUIs, and tangible or embodied data artefacts and installations.

\subsection{Limitations}

The main limitation of our work concerns our sample. Besides of having only eight participants, most of these had a Computer Science background. Since Computer Science students are used to working with data, but not with materials, this has probably influenced our findings. Although our observations held for the Product Design and Media Art students as well, our findings and insights regarding physicalisation literacy might mostly be relevant for people who are data literate and that are not designers or artists. Nevertheless, Computer Science students are a particularly challenging audience when engaging with materiality, as such this might be considered a strength as well.

Moreover, only four of eight participants agreed to be interviewed. Although interviews were based on an initial thematic analysis of all eight reports, the views of these four participants further fine-tuned our findings. Thus, further research should be conducted with a larger and more diverse sample of learners, in order to deepen and validate our initial findings.

Lastly and naturally, the choice and design of Data Diary assignments has influenced the process and thus our findings. For example, P3 indicated that the fourth Data Diary (senses and sensations) made them choose a subjective data source, as they saw the topic as subjective. Within a semester project, we were limited to five Data Diaries, and giving out an even wider range of exercises might yield even deeper observation and improve the process of learning, as well as providing more insight on the specific contribution of different kinds of tasks for the Data Diaries.

\section{CONCLUSION}

Prior work has mostly focused on the effect of physicalisation on data understanding and engagement with data [15, 23, 31]. Only little research (e.g. [22, 25, 57]) investigated the process of creating physicalisations and how to teach this. In this paper, we discussed the role of the Data Diaries in teaching physicalisation based on everyday data to participants who are used to visual data representations, but most of whom did not have designerly sensitivity.
Through an analysis of the Data Diaries, we show that participants went through four stages in which they learned how to represent data using materials. These four stages are: 1) participants feel overwhelmed by the idea, 2) they apply their knowledge of visualisation to create physicalisations, 3) they realise this does not work and discover that anything could be used to represent data, and 4) they realise that there should be a link between the data, method of collection, material, and story which you want to tell through the physicalisation. Besides materiality, it appears that even when being asked to create physicalisations, the visual remains dominant (in particular for Computer Science educated participants), with most of the resulting designs adhering to a 'view from afar' which ensures that the data can be understood by looking at it, without having to actively explore its 3D nature.

Furthermore, we discuss how creating physicalisations differs from visualisation, and how these differences force us to expand our notion of data. Compared to visualisations, it was impossible to represent data efficiently, objectively, and highly detailed. Instead, physicalisations linked back to the origin of the data, blurred the line between quantitative and qualitative, and focused on a connection between user and data. Therefore, the creation of physicalisations demands a broadened notion of what data is and at the same time facilitates this change or evolution in thinking. Thus, physicalisations may constitute a didactic vehicle for broadening learner's perspective of 'what is data'.

\section{ACKNOWLEDGMENTS}

We would like to thank our students Tahmina Arab, Julia Wetterling, Ludwig Lorenz, and the other five who wished to remain anonymous, for their hard work, contributions, and creativity throughout the project. This work was conducted on a grant from the Deutsche Forschungsgemeinschaft (DFG, German Research Foundation) -420584617 / HO3901/4-1.

\section{REFERENCES}

[1] Jason Alexander, Anne Roudaut, Jürgen Steimle, Kasper Hornbæk, Miguel Bruns Alonso, Sean Follmer, and Timothy Merritt. 2018. Grand Challenges in Shape-Changing Interface Research. In Proceedings of the 2018 CHI Conference on Human Factors in Computing Systems (Montreal QC, Canada) (CHI '18). Association for Computing Machinery, New York, NY, USA, 1-14. https: //doi.org/10.1145/3173574.3173873

[2] Basak Alper, Nathalie Henry Riche, Fanny Chevalier, Jeremy Boy, and Metin Sezgin. 2017. Visualization Literacy at Elementary School. In Proceedings of the 2017 CHI Conference on Human Factors in Computing Systems (CHI '17). Association for Computing Machinery, New York, NY, USA, 5485-5497. https: //doi.org/10.1145/3025453.3025877

[3] Stephen Barrass. 2016. Diagnosing Blood Pressure with Acoustic Sonification Singing Bowls. Int. 7. Hum.-Comput. Stud. 85, C (Jan. 2016), 68-71. https: //doi.org/10.1016/j.ijhcs.2015.08.007

[4] Jacques Bertin. 1983. Semiology of Graphics: Diagrams, Networks, Maps. University of Wisconsin Press, Madison.

[5] Jeremy Boy, Ronald A. Rensink, Enrico Bertini, and Jean-Daniel Fekete. 2014. A Principled Way of Assessing Visualization Literacy. IEEE Transactions on Visualization and Computer Graphics 20, 12 (Dec. 2014), 1963-1972. https://doi. org/10.1109/TVCG.2014.2346984

[6] Virginia Braun and Victoria Clarke. 2006. Using thematic analysis in psychology. Qualitative Research in Psychology 3, 2 (Jan. 2006), 77-101. https://doi.org/10. 1191/1478088706qp063oa

[7] Katy Börner, Andreas Bueckle, and Michael Ginda. 2019. Data visualization literacy: Definitions, conceptual frameworks, exercises, and assessments. Proceedings of the National Academy of Sciences 116, 6 (Feb. 2019), 1857-1864. https://doi.org/10.1073/pnas.1807180116

[8] Fanny Chevalier, Nathalie Henry Riche, Basak Alper, Catherine Plaisant, Jeremy Boy, and Niklas Elmqvist. 2018. Observations and Reflections on Visualization 
Literacy in Elementary School. IEEE Computer Graphics and Applications 38, 3 (May 2018), 21-29. https://doi.org/10.1109/MCG.2018.032421650

[9] Nick Couldry and Alison Powell. 2014. Big Data from the bottom up. Big Data \& Society 1, 2 (July 2014), $205395171453927 . \quad$ https://doi.org/10.1177/ 2053951714539277

[10] Audrey Desjardins and Heidi R. Biggs. 2021. Data Epics: Embarking on Literary Journeys of Home Internet of Things Data. In Proceedings of the $2021 \mathrm{CHI}$ Conference on Human Factors in Computing Systems. ACM, Yokohama, Japan, 1-17. https://doi.org/10.1145/3411764.3445241

[11] Audrey Desjardins and Timea Tihanyi. 2019. ListeningCups: A Case of Data Tactility and Data Stories. In Proceedings of the 2019 on Designing Interactive Systems Conference. ACM, San Diego CA USA, 147-160. https://doi.org/10.1145/ 3322276.3323694

[12] Catherine D'Ignazio. 2017. Creative data literacy: Bridging the gap between the data-haves and data-have nots. Information Design fournal 23 (July 2017), 6-18. https://doi.org/10.1075/idj.23.1.03dig

[13] Catherine D'Ignazio and Lauren F. Klein. 2020. Data Feminism. The MIT Press, Cambridge, Massachusetts, USA. https://mitpress.mit.edu/books/data-feminism

[14] Paul Dourish. 2017. The Stuff of Bits - An Essay on the Materialities of Information The MIT Press, Cambridge, MA. https://mitpress.mit.edu/books/stuff-bits

[15] Sean Follmer, Daniel Leithinger, Alex Olwal, Akimitsu Hogge, and Hiroshi Ishii 2013. inFORM: dynamic physical affordances and constraints through shape and object actuation. In Proceedings of the 26th annual ACM symposium on User interface software and technology. Association for Computing Machinery, New York, NY, USA, 417-426. https://doi.org/10.1145/2501988.2502032

[16] Vaike Fors, Åsa Bäckström, and Sarah Pink. 2012. Multisensory Emplaced Learning: Resituating Situated Learning in a Moving World. Moving World, Mind, Culture, and Activity 20, 2 (Aug. 2012), 170-183. https://doi.org/10.1080/10749039. 2012.719991

[17] Lisa Gitelman (Ed.). 2013. "Raw Data" Is an Oxymoron. The MIT Press, Cambridge, Massachusetts, USA. https://mitpress.mit.edu/books/raw-data-oxymoron

[18] Johannes Gäbler, Christoph Winkler, Nóra Lengyel, Wolfgang Aigner, Christina Stoiber, Günter Wallner, and Simone Kriglstein. 2019. Diagram Safari: A Visualization Literacy Game for Young Children. In Extended Abstracts of the Annual Symposium on Computer-Human Interaction in Play Companion Extended $A b-$ stracts (CHI PLAY '19 Extended Abstracts). Association for Computing Machinery, New York, NY, USA, 389-396. https://doi.org/10.1145/3341215.3356283

[19] Samantha Hautea, Sayamindu Dasgupta, and Benjamin Mako Hill. 2017. Youth Perspectives on Critical Data Literacies. In Proceedings of the 2017 CHI Conference on Human Factors in Computing Systems (CHI '17). Association for Computing Machinery, New York, NY, USA, 919-930. https://doi.org/10.1145/3025453.3025823

[20] Trevor Hogan. 2018. Data Sensification: beyond representation modality, toward encoding data in experience. In Design as a catalyst for change - DRS International Conference. Limerick, 1-15. https://doi.org/10.21606/drs.2018.238

[21] Trevor Hogan, Uta Hinrichs, Yvonne Jansen, Samuel Huron, Pauline Gourlet, Eva Hornecker, and Bettina Nissen. 2017. Pedagogy \& Physicalization: Designing Learning Activities around Physical Data Representations. In Proceedings of the 2017 ACM Conference Companion Publication on Designing Interactive Systems. ACM, Edinburgh United Kingdom, 345-347. https://doi.org/10.1145/3064857. 3064859

[22] Trevor Hogan and Eva Hornecker. 2012. How Does Representation Modality Affect User-Experience of Data Artifacts?. In Haptic and Audio Interaction Design (Lecture Notes in Computer Science), Charlotte Magnusson, Delphine Szymczak, and Stephen Brewster (Eds.). Springer, Berlin, Heidelberg, 141-151. https://doi org/10.1007/978-3-642-32796-4 15

[23] Trevor Hogan and Eva Hornecker. 2013. Blending the repertory grid technique with focus groups to reveal rich design relevant insight. In Proceedings of the 6th International Conference on Designing Pleasurable Products and Interfaces (DPPI '13). Association for Computing Machinery, New York, NY, USA, 116-125. https://doi.org/10.1145/2513506.2513519

[24] Sun-ha Hong. 2020. Technologies of Speculation -The Limits of Knowledge in a Data-Driven Society. NYU Press, New York.

[25] Samuel Huron, Pauline Gourlet, Uta Hinrichs, Trevor Hogan, and Yvonne Jansen 2017. Let's Get Physical: Promoting Data Physicalization in Workshop Formats In Proceedings of the 2017 Conference on Designing Interactive Systems. ACM, Edinburgh United Kingdom, 1409-1422. https://doi.org/10.1145/3064663.3064798

[26] Tim Ingold. 2011. The Perception of the Environment: Essays on Livelihood, Dwelling and Skill. Routledge, London.

[27] Hiroshi Ishii, Dávid Lakatos, Leonardo Bonanni, and Jean-Baptiste Labrune. 2012. Radical Atoms: Beyond Tangible Bits, toward Transformable Materials. Interactions 19, 1 (Jan. 2012), 38-51. https://doi.org/10.1145/2065327.2065337

[28] Yvonne Jansen, Pierre Dragicevic, Petra Isenberg, Jason Alexander, Abhijit Karnik, Johan Kildal, Sriram Subramanian, and Kasper Hornbæk. 2015. Opportunities and Challenges for Data Physicalization. In Proceedings of the 33rd Annual ACM Conference on Human Factors in Computing Systems (CHI '15). Association for Computing Machinery, New York, NY, USA, 3227-3236. https://doi.org/10.1145/ 2702123.2702180
[29] Britney Johnson, Ben Rydal Shapiro, Betsy DiSalvo, Annabel Rothschild, and Carl DiSalvo. 2021. Exploring Approaches to Data Literacy Through a Critical Race Theory Perspective. In Proceedings of the 2021 CHI Conference on Human Factors in Computing Systems. ACM, Yokohama Japan, 1-15. https://doi.org/10. 1145/3411764.3445141

[30] Maria Karyda, Merja Ryöppy, Jacob Buur, and Andrés Lucero. 2020. Imagining Data-Objects for Reflective Self-Tracking. In Proceedings of the $2020 \mathrm{CHI}$ Conference on Human Factors in Computing Systems. ACM, Honolulu HI USA, 1-12. https://doi.org/10.1145/3313831.3376844

[31] Maria Karyda, Danielle Wilde, and Mette Gislev Kjarsgaard. 2021. Narrative Physicalization: Supporting Interactive Engagement With Personal Data. IEEE Computer Graphics and Applications 41, 1 (Jan. 2021), 74-86. https://doi.org/10. 1109/MCG.2020.3025078

[32] Hellen Kennedy. 2018. Living with Data: Aligning Data Studies and Data Activism Through a Focus on Everyday Experiences of Datafication. Fournal for Contemporary Philosophy 1 (2018), 18-30.

[33] Helen Kennedy, Rosemary Lucy Hill, Giorgia Aiello, and William Allen. 2016. The work that visualisation conventions do. Information, Communication \& Society 19, 6 (June 2016), 715-735. https://doi.org/10.1080/1369118X.2016.1153126

[34] Melissa K. Kjelvik and Elizabeth H. Schultheis. 2019. Getting Messy with Authentic Data: Exploring the Potential of Using Data from Scientific Research to Support Student Data Literacy. CBE Life Sciences Education 18, 2 (2019), es2. https://doi.org/10.1187/cbe.18-02-0023

[35] Mary Anne Kuebel (Ed.). 2002. Living Learning: A Reader in Theme-Centered Interaction. Society for Media and Value Education, Delhi.

[36] Victor R. Lee. 2018. Personal Analytics Explorations to Support Youth Learning. In Digital Technologies and Instructional Design for Personalized Learning. IGI Global, Hershey, PA, 145-163. https://doi.org/10.4018/978-1-5225-3940-7.ch007

[37] Haiying Li, Janice Gobert, and Rachel Dickler. 2019. Scaffolding during Science Inquiry. In Proceedings of the Sixth (2019) ACM Conference on Learning @ Scale. ACM, Chicago IL USA, 1-10. https://doi.org/10.1145/3330430.3333628

[38] Giorgia Lupi and Stefanie Posavec. 2016. Dear Data. Particular Books / Penguin, London. http://giorgialupi.com/dear-data-2

[39] Deborah Lupton. 2017. Feeling your data: Touch and making sense of personal digital data. New Media \& Society 19, 10 (July 2017), 1599-1614.

[40] Deborah Lupton. 2018. How do data come to matter? Living and becoming with personal data. Big Data \& Society 5, 2 (July 2018), 205395171878631. https: //doi.org/10.1177/2053951718786314

[41] Jennifer Mankoff, Anind K. Dey, Gary Hsieh, Julie Kientz, Scott Lederer, and Morgan Ames. 2003. Heuristic Evaluation of Ambient Displays. In Proceedings of the SIGCHI Conference on Human Factors in Computing Systems (Ft. Lauderdale, Florida, USA) (CHI '03). Association for Computing Machinery, New York, NY, USA, 169-176. https://doi.org/10.1145/642611.642642

[42] Paul Marshall. 2007. Do Tangible Interfaces Enhance Learning?. In Proceedings of the 1st International Conference on Tangible and Embedded Interaction (Baton Rouge, Louisiana) (TEI '07). Association for Computing Machinery, New York, NY, USA, 163-170. https://doi.org/10.1145/1226969.1227004

[43] Nicole D. Martin, Catherine Dornfeld Tissenbaum, Dana Gnesdilow, and Sadhana Puntambekar. 2019. Fading distributed scaffolds: the importance of complementarity between teacher and material scaffolds. Instructional Science 47, 1 (Feb. 2019), 69-98. https://doi.org/10.1007/s11251-018-9474-0

[44] Viktor Mayer-Schönberger and Kenneth Cukier. 2014. Big Data: A Revolution That Will Transform How We Live, Work, and Think. Eamon Dolan/Mariner Books, Boston, Massachusetts, USA.

[45] Andrew Vande Moere. 2008. Beyond the Tyranny of the Pixel: Exploring the Physicality of Information Visualization. In 2008 12th International Conference Information Visualisation. 469-474. https://doi.org/10.1109/IV.2008.84

[46] Dietmar Offenhuber. 2019. Data by Proxy - Material Traces as Autographic Visualizations. IEEE Transactions on Visualization and Computer Graphics 26, 1 (July 2019), 98-108. https://doi.org/10.1109/TVCG.2019.2934788

[47] Dietmar Offenhuber. 2020. What we talk about when we talk about data physicality. IEEE Computer Graphics and Applications 40, 6 (June 2020), 25-37. https://doi.org/10.1109/MCG.2020.3024146

[48] Dietmar Offenhuber and Orkan Telhan. 2015. Indexical Visualization-The DataLess Information Display. In Ubiquitous Computing, Complexity, and Culture. Routledge, London, 288-303. https://doi.org/10.4324/9781315781129-31

[49] Luci Pangrazio and Neil Selwyn. 2019. 'Personal data literacies': A critical literacies approach to enhancing understandings of personal digital data. New Media \& Society 21, 2 (Feb. 2019), 419-437. https://doi.org/10.1177/1461444818799523

[50] Michael A Sao Pedro and Janice D Gobert. 2013. Incorporating Scaffolding and Tutor Context into Bayesian Knowledge Tracing to Predict Inquiry Skill Acquisition. In 6th International Conference on Educational Data Mining. The International Educational Data Mining Society, Massachusetts, 8.

[51] Theodore M. Porter. 1995. Trust in Numbers: The Pursuit of Objectivity in Science and Public Life. Princeton University Press, Princeton.

[52] Majken K. Rasmussen, Esben W. Pedersen, Marianne G. Petersen, and Kasper Hornbæk. 2012. Shape-Changing Interfaces: A Review of the Design Space and Open Research Questions. In Proceedings of the SIGCHI Conference on Human 
Factors in Computing Systems (Austin, Texas, USA) (CHI '12). Association for Computing Machinery, New York, NY, USA, 735-744. https://doi.org/10.1145/ 2207676.2207781

[53] Orit Shaer and Eva Hornecker. 2010. Tangible User Interfaces: Past, Present, and Future Directions. Foundations and Trends ${ }^{\circledR}$ in Human-Computer Interaction 3, 1-2 (2010), 4-137. https://doi.org/10.1561/1100000026

[54] Ben Rydal Shapiro, Amanda Meng, Cody O’Donnell, Charlotte Lou, Edwin Zhao, Bianca Dankwa, and Andrew Hostetler. 2020. Re-Shape: A Method to Teach Data Ethics for Data Science Education. In Proceedings of the 2020 CHI Conference on Human Factors in Computing Systems (CHI '20). Association for Computing Machinery, New York, NY, USA, 1-13. https://doi.org/10.1145/3313831.3376251

[55] Ricardo Sosa, Victoria Gerrard, Antonio Esparza, Rebeca Torres, and Robbie Napper. 2018. Data objects: Design principles for data physicalisation. In DS 92: Proceedings of the DESIGN 2018 15th International Design Conference. Faculty of Mechanical Engineering and Naval Architecture, University of Zagreb, The Design Society, Glasgow, 1685-1696.

[56] Faisal Taher, John Hardy, Abhijit Karnik, Christian Weichel, Yvonne Jansen, Kasper Hornbæk, and Jason Alexander. 2015. Exploring Interactions with Physically Dynamic Bar Charts. In Proceedings of the 33rd Annual ACM Conference on Human Factors in Computing Systems (CHI '15). Association for Computing Machinery, New York, NY, USA, 3237-3246. https://doi.org/10.1145/2702123. 2702604

[57] Alice Thudt, Uta Hinrichs, Samuel Huron, and Sheelagh Carpendale. 2018. SelfReflection and Personal Physicalization Construction. In Proceedings of the 2018 CHI Conference on Human Factors in Computing Systems. ACM, Montreal QC Canada, 1-13. https://doi.org/10.1145/3173574.3173728

[58] Sherry Turkle (Ed.). 2007. Evocative Objects: Things We Think With. MIT Press, Cambridge, MA, USA.

[59] B. Ullmer and H. Ishii. 2000. Emerging Frameworks for Tangible User Interfaces. IBM Syst. 7. 39, 3-4 (July 2000), 915-931. https://doi.org/10.1147/sj.393.0915

[60] Lev S. Vygotsky. 1978. Mind in Society: Development of Higher Psychological Processes. Harvard University Press, Cambridge, MA. https://doi.org/10.2307/j. ctvjf9vz4
[61] Hannes Waldschütz and Eva Hornecker. 2020. The Importance of Data Curation for Data Physicalization. In Companion Publication of the 2020 ACM Designing Interactive Systems Conference (DIS'20 Companion). Association for Computing Machinery, New York, NY, USA, 293-297. https://doi.org/10.1145/3393914.3395892

[62] Yun Wang, Adrien Segal, Roberta Klatzky, Daniel F. Keefe, Petra Isenberg, Jorn Hurtienne, Eva Hornecker, Tim Dwyer, Stephen Barrass, and Theresa-Marie Rhyne. 2019. An Emotional Response to the Value of Visualization. IEEE Computer Graphics and Applications 39, 5 (Sept. 2019), 8-17. https://doi.org/10.1109/MCG. 2019.2923483

[63] Zezhong Wang, Lovisa Sundin, Dave Murray-Rust, and Benjamin Bach. 2020. Cheat Sheets for Data Visualization Techniques. In Proceedings of the $2020 \mathrm{CHI}$ Conference on Human Factors in Computing Systems (CHI '20). Association for Computing Machinery, New York, NY, USA, 1-13. https://doi.org/10.1145/ 3313831.3376271

[64] Data Physicalization Wiki. 2021. Teaching - Data Physicalization. http:// dataphys.org/wiki/Teaching

[65] Wesley Willett, Yvonne Jansen, and Pierre Dragicevic. 2017. Embedded Data Representations. IEEE Transactions on Visualization and Computer Graphics 23, (Jan. 2017), 461-470. https://doi.org/10.1109/TVCG.2016.2598608 Conference Name: IEEE Transactions on Visualization and Computer Graphics.

[66] Annika Wolff, Daniel Gooch, Jose J. Cavero Montaner, Umar Rashid, and Gerd Kortuem. 2016. Creating an Understanding of Data Literacy for a Data-driven Society. The fournal of Community Informatics 12, 3 (Aug. 2016), 9-26. https: //doi.org/10.15353/joci.v12i3.3275

[67] Delia Yi Min Lim, Christine Ee Ling Yap, and Jung-Joo Lee. 2021. Datastorming: Crafting Data into Design Materials for Design Students' Creative Data Literacy. In Creativity and Cognition. ACM, Virtual Event Italy, 1-9. https://doi.org/10. 1145/3450741.3465246

[68] Oren Zuckerman, Saeed Arida, and Mitchel Resnick. 2005. Extending Tangible Interfaces for Education: Digital Montessori-Inspired Manipulatives. In Proceedings of the SIGCHI Conference on Human Factors in Computing Systems (Portland, Oregon, USA) (CHI '05). Association for Computing Machinery, New York, NY, USA, 859-868. https://doi.org/10.1145/1054972.1055093 


\section{A TEACHING CURRICULUM}

Table 3: Overview of the given presentations, homework, and mandatory readings.

\begin{tabular}{|c|c|c|c|}
\hline Week & Presentations On & Homework & Readings \\
\hline 1 & Introduction to data physicalisations & $\begin{array}{l}\text { Students had to present themselves using } \\
\text { statistics and a data physicalisation which } \\
\text { they liked }\end{array}$ & [45] and [27] \\
\hline 2 & $\begin{array}{l}\text { Presenting and collecting data, and } \\
\text { Visualisation principles, affordances, and } \\
\text { modalities }\end{array}$ & Data Diary 1 & [52] and [28] \\
\hline 3 & Materiality and data & Data Diary 2 & {$[30]$} \\
\hline 4 & Data and ethics & $\begin{array}{l}\text { Data Diary } 3 \text { and students presented their } \\
\text { initial three ideas for their final data physi- } \\
\text { calisation }\end{array}$ & [55] and [61] \\
\hline 5 & Prototyping and design processes, part 1 & Data Diary 4 & {$[1]$} \\
\hline 6 & Prototyping and design processes, part 2 & $\begin{array}{l}\text { Data Diary } 5 \text { and students pitched their con- } \\
\text { cept for the final data physicalisation }\end{array}$ & [31] \\
\hline $7-10$ & Feedback session and electronics tutorial & Students presented their progress & \\
\hline 11 & How to write a report & Students presented their progress & \\
\hline 12 & & $\begin{array}{l}\text { Final Presentation: Students presented their } \\
\text { data physicalisation }\end{array}$ & \\
\hline
\end{tabular}




\section{B DEVELOPMENT OVERVIEW WITHIN THE DATA DIARIES}

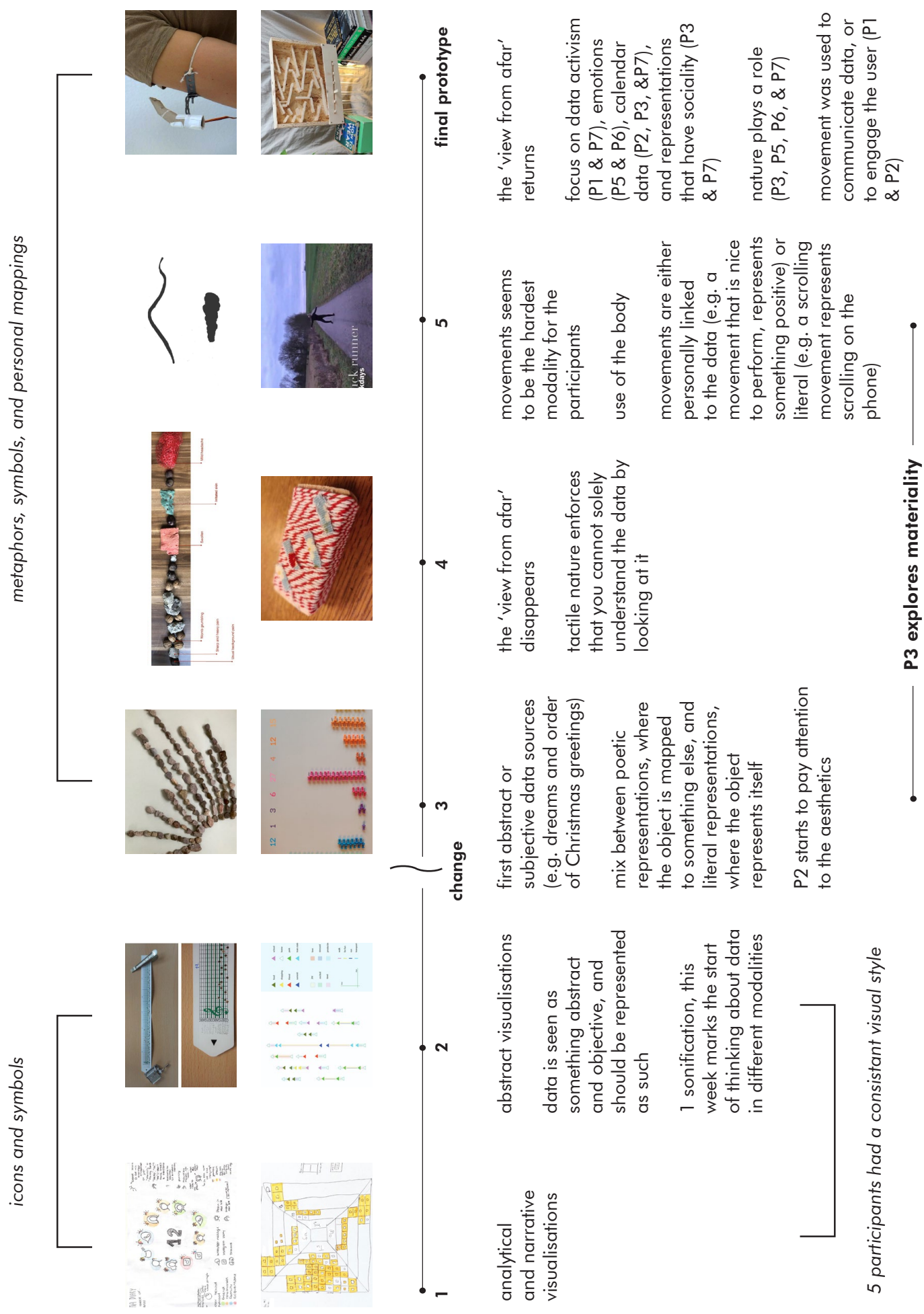

Figure 10: An overview of the created physicalisations and the changes in perspective, style and insights noticeable in students' designs and reports. 


\section{FIGURE DESCRIPTIONS FIGURE 9 AND 10}

Table 4: Figure descriptions of Figure 9 and 10.

\begin{tabular}{|c|c|}
\hline Figure & Alt Text \\
\hline 9 & 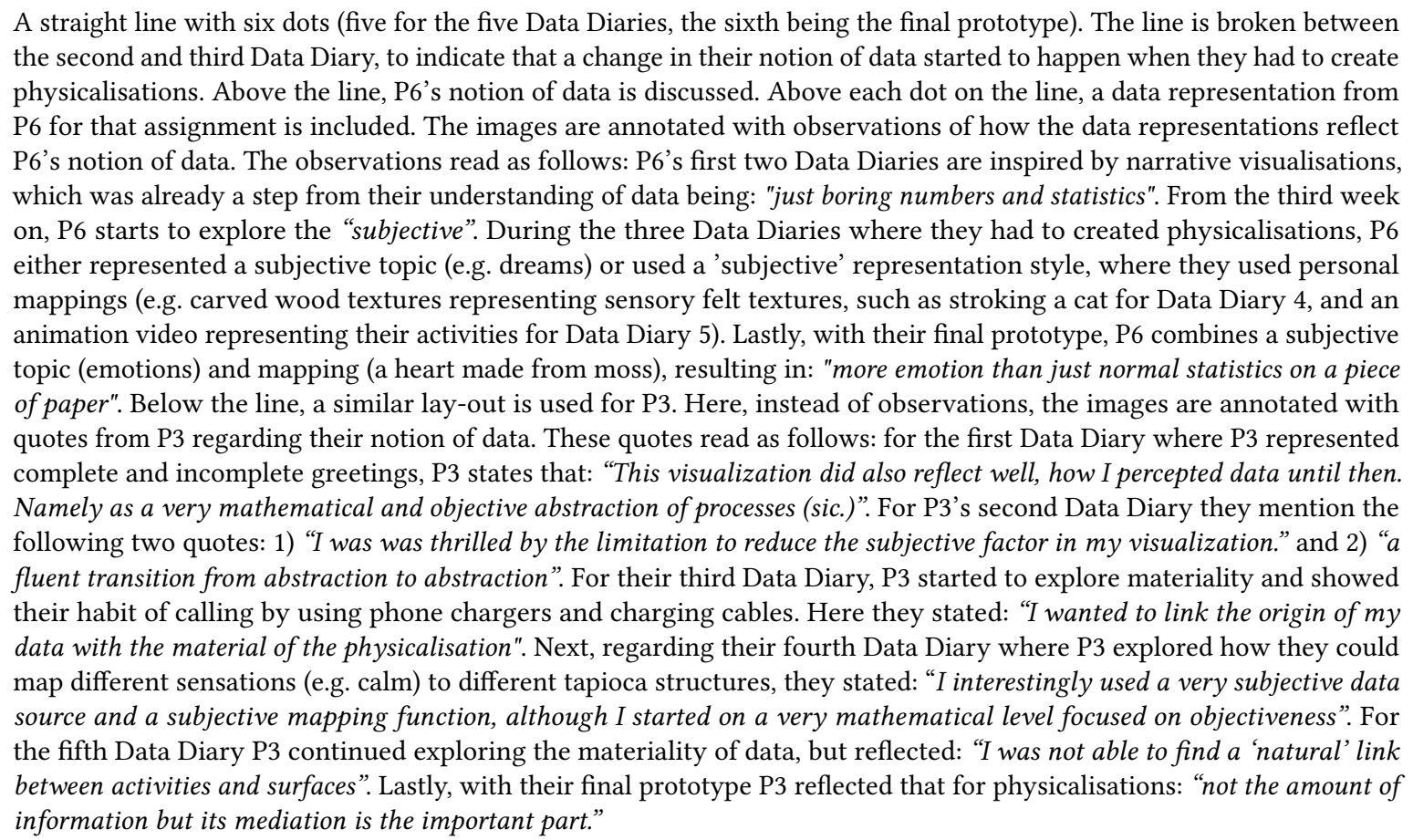 \\
\hline 10 & $\begin{array}{l}\text { A straight line with six dots. The dots are labelled 1-5 (representing the Data Diaries). The sixth dot is the final prototype. } \\
\text { Above the line are two images per Data Diary and final prototype. These have been discussed in the previous sections. } \\
\text { The first two Data Diaries have the note that they used icons and symbols, and five participants kept a similar visual style } \\
\text { in these assignments. The other Data Diaries and final prototype have the note that they relied on metaphors, symbols, } \\
\text { and personal mappings. The line is broken between Data Diary } 2 \text { and 3, indicating changes once the physicalisation } \\
\text { started. Below the line are comments on the developments, style, or insights which stood out for each assignment. These } \\
\text { read as follows: Data Diary } 1 \text { - analytical and narrative visualisations. Data Diary } 2 \text { - abstract visualisations; data is seen } \\
\text { as something abstract and objective, and should be represented as such; } 1 \text { sonification, this week marks the start of } \\
\text { thinking about data in different modalities. Data Diary } 3 \text { - first abstract or subjective data sources (e.g. dreams and order } \\
\text { of Christmas greetings); mix between poetic representations, where the object is mapped to something else, and literal } \\
\text { representations, where the object represents itself; P2 starts to pay attention to the aesthetics. Data Diary } 4 \text { - the 'view } \\
\text { from afar' disappears; the tactile nature enforces that you cannot solely understand the data by looking at it. Data Diary } 5 \\
\text { - movements seems to be the hardest modality for the participants. They often resort to created movements with their } \\
\text { body. Movements are either personally linked to the data (e.g. a movement that is nice to perform, represents something } \\
\text { positive) or literal (e.g. a scrolling movement represents scrolling on the phone). Final prototype - the 'view from afar' } \\
\text { returns; focus on data activism (P1 + P7), emotions (P5 +P6), calendar data (P2, P3, + P7), and representations that have } \\
\text { sociality (P3 + P7); nature plays a role (P3, P5, P6, + P7); movement was used to communicate data, or to engage the user } \\
\text { (P1 + P2). Finally, the images states that with Data Diary } 1 \text { and 2, } 5 \text { participants had a consistent visual style, and that P3 } \\
\text { explored materiality in Data Diary 3, 4, and 5. }\end{array}$ \\
\hline
\end{tabular}

